\title{
Response of an oscillator to a random quadratic velocity-feedback loading
}

\author{
Vincent Denoël, Luigi Carassale
}

\begin{abstract}
The Aerodynamic force acting on compact structures is often modeled as a quadratic function of the wind velocity, which fluctuates randomly due to the atmospheric turbulence. When a flexible structure is considered and the quasi-steady assumption is applied, the wind velocity is substituted by the wind-structure relative velocity and, even in case of linear structures, the composite aerodynamic-mechanical system is governed by a nonlinear differential equation characterized by a quadratic feedback term. This class of dynamical systems has been deeply investigated (with different levels of simplifications) applying several alternative mathematical approaches. In this paper we define a system approximation based on a 2nd-order Volterra series and obtain its statistical response in terms of cumulants. The response cumulants are calculated applying the Multiple Scale Spectral Analysis leading to analytic or semi-analytic expressions. All the approximations are validated through Monte Carlo simulation within a wide parameter space. Then, the analytical structure of the obtained expressions is used to discuss, from a qualitative point of view, the behavior of the considered dynamical system.
\end{abstract}

\section{Introduction}

In several engineering problems, the driving force is expressed as a nonlinear transformation of the input, the response and its time derivatives. In structural engineering, this is the case in vibrations of base-excited structures, where the internal forces depend on the (nonlinear transformation of) relative displacements and velocities between the structure and the base motion [Constantinou and Papageorgiou, 1990. Other examples include the aerodynamic or hydrodynamic loads on flexible structures, where the effective loads might be expressed, in a quasi-steady framework, as a memoryless nonlinear transformation of some relative velocities (e.g. Kareem, 1987). The common feature shared by these models with nonlinear feedback is the presence of random parametric excitation terms. The nonlinearity associated with these terms, as well as other more specific terms (like the quadratic structural velocity term considered in this paper) make the development of exact analytical solutions rather challenging.

In this paper we consider the problem of a single degree-of-freedom linear oscillator, subject to a quasi-steady aerodynamic loading. The aerodynamic force is defined as the square of the wind-structure relative velocity and results from the sum of five terms: a constant term, terms proportional to the turbulence fluctuation and its square, terms proportional to the structure velocity and its square and a term proportional to the product of wind velocity fluctuation and structure velocity. Another specificity of this problem concerns the stochasticity of the wind velocity fluctuation, which is usually modeled as a zero-mean, stationary, Gaussian random process. The need to regard this problem from a probabilistic viewpoint makes it even more difficult to develop closed-form solutions. Of course Monte Carlo simulations are able to deal with the matter (e.g. Gurley et al., 1997, Di Paola, 1998), but they do not offer the same understanding of the problem as a closed-form expression.

The seminal works related to this problem are those of Davenport 1961 who disregarded the nonlinear components of the loading, for the sake of simplicity, or perhaps, as only the standard deviation of the response was of interest. Later, the effects of the quadratic term of the wind velocity fluctuation, and consequently the nonGaussianity of the excitation, was deeply investigated by several authors mostly in the 1980's and 1990's. SSoize 1978 demonstrated that the Gaussian-input approximation could be removed obtaining the joint characteristic function of the structure response and velocity, and hence the peak value of the response. Grigoriu and co-workers (Grigoriu, 1986, Grigoriu and Ariaratnam, 1988, Buss and Grigoriu, 1990) followed the same line exploring a number of mathematical strategies to tackle the problem, including a different type of characteristic function method, the moment equations derived through the Itô calculus and the path-integral method. The potentials of the moment equation method was deeply investigated by Benfratello et al. [1996], Gullo et al. [1998, Benfratello and Muscolino [1999], who extended the application to the case of multi-dof wind-excited structures and [Floris et al. [2001], who included the structure velocity feedback in the picture. The use of the Itô calculus and the moment equations, in particular, has two important drawbacks. As a basic hypothesis, the external excitation must be white, thus 
the reproduction of a realistic atmospheric turbulence requires the realization of a pre-filter and the addition of fictitious degrees of freedom or the use of an augmented state vector |Benfratello and Muscolino, 1999. On the other hand, the presence of the structure velocity feedback produces relationships among the moment equations such that the statistical moment of order $j$ depend on statistical moments of orders greater than $j$. This structure of the equations, referred to open hierarchy, requires the definition of a closure scheme, which is usually realized in terms of cumulants Di Paola et al. 1992. In parallel to the mentioned contributions, different frequency-domain approaches have been applied (e.g.Kareem, 1984; Gusella and Materazzi, 2000) leading to expressions providing some statistical properties of the response. In this context, Kareem and Zhao [1994] borrowed from the offshoreengineering community the idea of using the Volterra series to represent the given nonlinear system retaining both the quadratic wind velocity fluctuation, as well as the nonlinear velocity feedback. The Volterra series was then employed by several authors (e.g. Benfratello et al. 1998, Kareem et al., 1998) demonstrating that a second order Volterra series reproduces accurately the given nonlinear system, at least for some specific choice of the parameters.

This short review indicates two facts: (i) the need to consider the non-Gaussianity of the loading, as it may significantly affect the peak values of the response, (ii) the absence of closed-form approximate solutions considering the nonlinear terms and able to provide a probabilistic characterization of the response. These issues are approached in this work with two arguments. First, several works have suggested that a second order Volterra model may provide an accurate modeling for the considered class of problems [Carassale and Kareem, 2010]. Second, as the timescales related to the wind fluctuations and the structure are well separated, the use of a perturbation approach is welcome. With this respect, the recent Multiple Timescale Spectral Analysis [Denoël, 2015] is substantially used in the formulation. The combination of these two arguments are validated through an extensive Monte Carlo simulation spanning a wide parameter space. The accuracy of the second order Volterra representation in validated comparing its response calculated through the Associated Linear Equations |Vazquez Feijoo et al., 2005 and the response of the given nonlinear system. Then, analytic and semi-analytic expressions for the cumulants of the response are derived and compared with the result of the Monte Carlo simulation. Once the approximated dynamical model and the approximated expressions of the response cumulants are validated, the role of the system parameters and the asymptotic behaviors of the system are analyzed and discussed.

\section{Position of the Problem}

\subsection{Governing equation}

Under the quasi-steady assumption, the response of a point-like single degree-of-freedom structure subject to a 1-dimensional wind turbulence is governed by the nonlinear second order differential equation (e.g. Kareem and Zhao [1994])

$$
m \ddot{x}+c \dot{x}+k x=\frac{1}{2} \rho A c_{d}(U+u-\dot{x})^{2}
$$

where $x(t)$ is the structural displacement, $m, c$ and $k$ are mass, viscosity and stiffness, respectively, $U$ is the mean wind velocity and $u(t)$ a Gaussian, zero-mean, stationary random process representing the wind velocity fluctuation; $\rho, A$ and $c_{d}$ are, respectively, the air density, the area of the structure exposed to the wind and the aerodynamic drag coefficient. The overhead dot denotes differentiation with respect to time $t$. A first nonlinearity of this equation results from the squared structural velocity $\dot{x}^{2}(t)$ obtained in the right-hand side after expansion. It is referred to as the quadratic structural velocity term. A second one is related to the random parametric excitation term proportional to $u(t) \dot{x}(t)$, which also arises in the right-hand side after expansion. These two nonlinearities typify the random quadratic velocity-feedback of the simple model of a wind-loaded structure. We demonstrate in this paper that the former nonlinearity is of second order for common turbulence intensities, whilst the latter may significantly affect the third and fourth cumulants of the response.

Lacking simple tools for the proper simulation of these two terms, but a full Monte Carlo simulation, they are generally neglected. The problem thus falls into linear structural dynamics involving however a quadratic transformation of the Gaussian input turbulence velocity which is responsible for the non Gaussianity of the loading. As far as the second order solution of this problem is considered, the quadratic term $u^{2}(t)$ is known to be of relatively weak importance, as was extensively investigated during the 1980's (e.g. Kareem, 1984). Accordingly, dropping the quadratic term $u^{2}(t)$ further simplifies the problem to a fully linear one

$$
m \ddot{x}+\left(c+\rho A c_{d} U\right) \dot{x}+k x=\frac{1}{2} \rho A c_{d}\left(U^{2}+2 U u\right)
$$


which then preserves the input-output Gaussianity. The Gaussian response $x(t)$ of this model is thus completely characterized by its Power Spectral Density (PSD) function $S_{x}(\omega)$, expressed as a function of the PSD of the wind velocity fluctuation $S_{u}(\omega)$. Several PSD models exist for $S_{u}(\omega)$ [Solari and Piccardo, 2001], with the common property that the frequency content of the turbulence decreases as $\omega^{-5 / 3}$ in the high-frequency range, a result of the famous Kolmogorov energy cascade. One such model reads

$$
S_{u}\left(\omega ; \frac{U}{L}, \sigma_{u}^{2}\right)=\sigma_{u}^{2} \frac{0.546 \frac{L}{U}}{\left(1+1.64 \frac{L}{U}|\omega|\right)^{5 / 3}}
$$

where $\sigma_{u}=I_{u} U$ is the standard deviation of $u$ and $I_{u}$ is the turbulence intensity, $\omega$ is the circular frequency and $L$ is the integral length scale of the turbulence. This is the model considered in the illustrations of this paper, although the developments presented next are valid for any wind turbulence spectrum. Notice that we use a semicolon to indicate additional parameters of the model; the list of parameters is usually dropped in the equations in order to ease readability.

\subsection{Dimensionless formulation}

This problem might be formulated in a dimensionless manner, with the introduction of the following characteristic response and time

$$
x^{\star}=I_{u} \frac{\rho A c_{d} U^{2}}{k} \quad ; \quad t^{\star}=\sqrt{\frac{m}{k}}=\frac{1}{\omega_{0}}
$$

representing the standard deviation of the linear quasi-static response, i.e. the quasi-static response predicted by (2), and the inverse of the natural circular frequency of the oscillator. The dimensionless response $\tilde{x}=x / x^{\star}$ and time $\tilde{t}=t / t^{\star}=\omega_{0} t$ are naturally introduced, together with the symbol ' related to differentiation with respect to this new time coordinate. A dimensionless, zero-mean and unit variance, wind velocity fluctuation $\tilde{u}=u / \sigma_{u}$ is also introduced. The corresponding PSD $S_{\tilde{u}}(\tilde{\omega} ; \alpha)$ is

$$
S_{\tilde{u}}(\tilde{\omega} ; \alpha)=\frac{\omega_{0}}{\sigma_{u}^{2}} S_{u}\left(\tilde{\omega} \omega_{0} ; \alpha \omega_{0}, \sigma_{u}^{2}\right)
$$

with $\tilde{\omega}=\omega / \omega_{0}$ being the dimensionless frequency parameter and $\alpha=\frac{U}{L \omega_{0}}$ the dimensionless characteristic frequency of the turbulence velocity. This latter number - relating thus the characteristic frequency of turbulence $U / L$ to the characteristic frequency $\omega_{0}$ of the structure - is typically small in wind engineering applications, in the range $\left[10^{-3} ; 10^{-1}\right]$, and offers the possible use of multiple timescales approaches such as the well-known background/resonant decomposition [Davenport, 1961] or its higher-order adaptation [Denoël, 2011, 2015. The normalization property of the PSD translates into

$$
\int_{-\infty}^{+\infty} S_{\tilde{u}}(\tilde{\omega} ; \alpha) d \tilde{\omega}=1
$$

regardless of the value of the parameter $\alpha$. Notice that $S_{\tilde{u}}(\tilde{\omega} ; \alpha)$ might be expressed as a function of additional parameters, if the original density $S_{u}(\omega)$ happens to be more complex than (3).

After division by $k x^{\star}$, the governing equation (1) becomes

$$
\tilde{x}^{\prime \prime}+2 \xi_{s} \tilde{x}^{\prime}+\tilde{x}=\frac{1}{2 I_{u}}\left(1+I_{u} \tilde{u}-2 I_{u} \xi_{a} \tilde{x}^{\prime}\right)^{2}
$$

where the additional coefficients

$$
\xi_{s}=\frac{c}{2 m \omega_{0}} \quad ; \quad \xi_{a}=\frac{\rho A c_{d} U}{2 m \omega_{0}}
$$

are readily identified as the structural and aerodynamic damping coefficients, respectively. After expanding the right-hand side and collecting together both sources of damping, the governing equation reads

$$
\tilde{x}^{\prime \prime}+2\left(\xi_{s}+\xi_{a}\right) \tilde{x}^{\prime}+\tilde{x}=\frac{1}{2 I_{u}}+\tilde{u}+\frac{I_{u}}{2} \tilde{u}^{2}-2 I_{u} \xi_{a} \tilde{u} \tilde{x}^{\prime}+2 I_{u} \xi_{a}^{2} \tilde{x}^{\prime 2}
$$

with $\xi=\xi_{s}+\xi_{a}$ the total damping coefficient, by definition. The solution of this equation has no known closed-form expression, but is formally written introducing the nonlinear operator $\mathcal{H}[\cdot]$ as

$$
\tilde{x}=\mathcal{H}\left[\tilde{u} ; \xi_{s}, \xi_{a}, I_{u}\right]
$$


in which the input $\tilde{u}$ is characterized by the PSD $S_{\tilde{u}}(\tilde{\omega} ; \alpha)$.

Interestingly enough, all four dimensionless numbers involved in this governing equation, $\left\{\alpha, I_{u}, \xi_{s}, \xi_{a}\right\}$ are small numbers. This property is extensively used in the sequel, in order to perform a closed form analytical study of this system, by means of a perturbation method.

\subsection{Solution Methodology}

The influence of the last two forcing terms in (9) has never been studied neither exhaustively nor in closed form. This is the objective of this study. Invoking the smallness of the last two forcing terms, compared to the turbulent loading term $\tilde{u}$, it is readily seen that these two terms affect only marginally the magnitude of the response. The second order response and the bulk of the distribution are thus well captured with a linear model. The main focus therefore consists in quantifying the influence of the nonlinear loading terms on higher statistical cumulants, in our case the third and fourth cumulants of the response.

In contrast with previous attempts at tackling this problem by means of the moment equations |Floris et al., 2001], the model is represented here as a second order Volterra model [Kareem and Zhao, 1994], which better suits the polynomial nonlinearity and the parametric nature of the nonlinear feedback. Apart from not being dependent on the choice of a closure technique as in the moment equation approaches, there is no big advantage in implementing the Volterra model straightforwardly, as it performs equally to crude Monte Carlo simulation in terms of accuracy and computational burden Carassale and Kareem, 2010, at least when one specific set of parameters is considered.

The most time-consuming step in the use of Volterra models is the calculation of the response cumulants, which is usually carried out by the numerical evaluation of multiple integrals in the frequency-domain [Li et al., 1995]. For second order systems, the integration of the frequency-response functions can be simplified by computing a suitable factorization |Kac and Siegert, 1947] or, by considering the existence of multiple timescales in the problem, as suggested by the smallness of parameters $\alpha$ and $\xi$. Extrapolating on this idea, we apply a recent method, the Multiple Timescale Spectral Analysis, for the approximation of the cumulants of the response of systems with multiple timescales [Denoël, 2015], to obtain closed-form expressions for the second, third and fourth cumulants of the response. This operation might be viewed as an extension of the well-known Background/Resonant decomposition technique that is commonly used for the second order response of linear oscillators [Davenport, 1961]. With these approximations in hand, we perform an exhaustive study of the closed-form response of the problem.

In order to provide a tangible insight onto the replacement of the nonlinear problem by its linearized counterpart (the approximation that was intensively adopted over the last decades), our results are also presented by comparison with the response of the linear system

$$
\tilde{x}_{\ell}^{\prime \prime}+2\left(\xi_{s}+\xi_{a}\right) \tilde{x}_{\ell}^{\prime}+\tilde{x}_{\ell}=\frac{1}{2 I_{u}}+\tilde{u}+\frac{I_{u}}{2} \tilde{u}^{2}
$$

which is also written with the linear operator $\mathcal{L}[\cdot]$ as

$$
\tilde{x}_{\ell}=\mathcal{L}\left[\tilde{u} ; \xi_{s}, \xi_{a}, I_{u}\right] .
$$

Comparison of (9) and (11) shows that the linear equation is recovered from the nonlinear one by setting the aerodynamic damping $\xi_{a}$ equal to zero, then replacing the structural damping by the sum of the structural and aerodynamic ones, i.e. formally $\mathcal{L}\left[\tilde{u} ; \xi_{s}, \xi_{a}, I_{u}\right]=\mathcal{H}\left[\tilde{u} ; \xi_{s}+\xi_{a}, 0, I_{u}\right]$.

The rest of the paper is organized as follows. In Section 3, the second order Volterra model of the problem is developed, together with its kernels and associated linear equations. The truncation of the Volterra model to the second order is also validated by comparison with Monte Carlo simulations of the nonlinear system (9). In Section 4, general developments are given for the PSD, bispectrum and trispectrum of the response of the second order Volterra model. Then, the timescale separation between the loading and the resonance properties of the oscillator are considered in order to provide approximations of the cumulants. These are then analyzed and discussed along Section 6 .

\section{The Second Order Volterra Model}

An approximate solution to the given nonlinear problem is derived by substituting the dynamical system defined by (9) with a second order Volterra series [Schetzen, 1980]. According to this idea, the response is written as a series

$$
\tilde{x}(\tilde{t})=\tilde{x}_{0}+\tilde{x}_{1}(\tilde{t})+\tilde{x}_{2}(\tilde{t})
$$


where the zeroth term $\tilde{x}_{0}$ is a constant and where the terms $\tilde{x}_{i}(\tilde{t})=\mathcal{H}_{i}[\tilde{u}], i=1,2$, result from the Volterra operators $\mathcal{H}_{i}$ applied to the normalized fluctuation $\tilde{u}$. They are defined as

$$
\tilde{x}_{1}(\tilde{t})=\int_{-\infty}^{+\infty} h_{1}(\tau) \tilde{u}(\tilde{t}-\tau) d \tau \quad ; \quad \tilde{x}_{2}(\tilde{t})=\iint_{-\infty}^{+\infty} h_{2}\left(\tau_{1}, \tau_{2}\right) \tilde{u}\left(\tilde{t}-\tau_{1}\right) \tilde{u}\left(\tilde{t}-\tau_{2}\right) d \tau_{1} d \tau_{2}
$$

where $h_{1}(\tau)$ and $h_{2}\left(\tau_{1}, \tau_{2}\right)$ represent the Volterra kernels of the problem. Former works Carassale and Kareem 2010] have suggested that the truncation of this series to the second order, retaining thus terms up to $\tilde{x}_{2}(t)$, is sufficient to represent the statistics of the considered problem up to the fourth cumulant. This assertion is validated in Section 3.4 with a much wider set of parameters than the single-parameter simulation Carassale and Kareem, 2010] that has inspired this work. The replacement of the actual nonlinear governing equation by a second order Volterra model is a cornerstone in these developments as it precisely allows the derivation of the explicit solutions presented in Section 4

\subsection{Volterra Frequency Response Functions}

The Volterra Frequency-Response Functions (VFRF) $H_{1}(\tilde{\omega})$ and $H_{2}\left(\tilde{\omega}_{1}, \tilde{\omega}_{2}\right)$ are defined as the multi-dimensional Fourier transforms of the kernels

$$
H_{1}(\tilde{\omega})=\int_{-\infty}^{+\infty} h(\tau) e^{-\mathrm{i} \tilde{\omega} \tau} d \tau \quad ; \quad H_{2}\left(\tilde{\omega}_{1}, \tilde{\omega}_{2}\right)=\iint_{-\infty}^{+\infty} h\left(\tau_{1}, \tau_{2}\right) e^{-\mathrm{i} \tilde{\omega}_{1} \tau_{1}-\mathrm{i} \tilde{\omega}_{2} \tau_{2}} d \tau_{1} d \tau_{2},
$$

while $H_{0}=\tilde{x}_{0}$ for completeness. For a given problem, they may be established with the harmonic probing technique |Bedrosian and Rice, 1971] or with the systematic procedure presented in Carassale and Kareem [2010]. Following the same steps as in this latter approach, which are reported in the Appendix A for clarity, the VFRFs of the considered problem are obtained as

$$
\begin{gathered}
H_{0}=\frac{1}{2 I_{u}}, \quad H_{1}(\tilde{\omega})=\frac{1}{\hat{D}(\tilde{\omega})}, \\
H_{2}\left(\tilde{\omega}_{1}, \tilde{\omega}_{2}\right)=\frac{I_{u}}{2} \frac{\left(1-2 \xi_{a} \mathrm{i} \tilde{\omega}_{1} H_{1}\left(\tilde{\omega}_{1}\right)\right)\left(1-2 \xi_{a} \mathrm{i} \tilde{\omega}_{2} H_{1}\left(\tilde{\omega}_{2}\right)\right)}{\hat{D}\left(\tilde{\omega}_{1}+\tilde{\omega}_{2}\right)}
\end{gathered}
$$

where $\hat{D}(\tilde{\omega})=1-\tilde{\omega}^{2}+2 \mathrm{i}\left(\xi_{s}+\xi_{a}\right) \tilde{\omega}$. These two functions are sketched in Fig. 1 for some specific values of the structural and aerodynamic damping coefficients. While the first one corresponds to the classical frequency response function of a linear oscillator, the second order frequency response function illustrates the interaction between the different harmonics in the response, especially the filtering of pairs of harmonics $\left(\tilde{\omega}_{1}, \tilde{\omega}_{2}\right)$ that fall out of the band $\left|\tilde{\omega}_{1}+\tilde{\omega}_{2}\right| \lesssim 1$. They feature the general symmetry properties $H_{1}(-\omega)=\bar{H}_{1}(\omega), H_{2}\left(\omega_{1}, \omega_{2}\right)=H_{2}\left(\omega_{2}, \omega_{1}\right)$ and $H_{2}\left(-\omega_{1},-\omega_{2}\right)=\bar{H}_{2}\left(\omega_{1}, \omega\right)$ of VFRFs (the overhead bar being the complex conjugate:Schetzen, 1980), that will prove helpful in the following developments.

The second order Volterra frequency response function $H_{2}$ models the non Gaussianity of the response. It is interesting to expand the expression of $\mathrm{H}_{2}$ to highlight its different contributions. Tracing back the developments in Appendix A, it readily appears that $H_{2}:=H_{2, u^{2}}+H_{2, u \dot{x}}+H_{2, \dot{x}^{2}}$ where

$$
\begin{aligned}
& H_{2, u^{2}}=\frac{I_{u}}{2} \frac{1}{\hat{D}\left(\tilde{\omega}_{1}+\tilde{\omega}_{2}\right)}, \\
& H_{2, u \dot{x}}=-I_{u} \xi_{a} \mathrm{i} \frac{\tilde{\omega}_{1} H_{1}\left(\tilde{\omega}_{1}\right)+\tilde{\omega}_{2} H_{1}\left(\tilde{\omega}_{2}\right)}{\hat{D}\left(\tilde{\omega}_{1}+\tilde{\omega}_{2}\right)}, \\
& H_{2, \dot{x}^{2}}=-2 I_{u} \xi_{a}^{2} \frac{\tilde{\omega}_{1} \tilde{\omega}_{2} H_{1}\left(\tilde{\omega}_{1}\right) H_{1}\left(\tilde{\omega}_{2}\right)}{\hat{D}\left(\tilde{\omega}_{1}+\tilde{\omega}_{2}\right)} .
\end{aligned}
$$

This formulation splits up the terms specifically related to the squared turbulence $\left(\sim \xi_{a}^{0}\right)$, the parametric excitation $\left(\sim \xi_{a}\right)$ and the quadratic structural velocity $\left(\sim \xi_{a}^{2}\right)$. If the linear governing equation (11) is considered instead of the nonlinear governing equation (9), i.e. setting $\xi_{a}=0$ or dropping thus the nonlinear terms $u \dot{x}$ and $\dot{x}^{2}$ of the loading, the second Volterra frequency response function boils down to $H_{2, u^{2}}$ instead of the complete expression (17) and provides the exact representation of of the system defined by (11). 

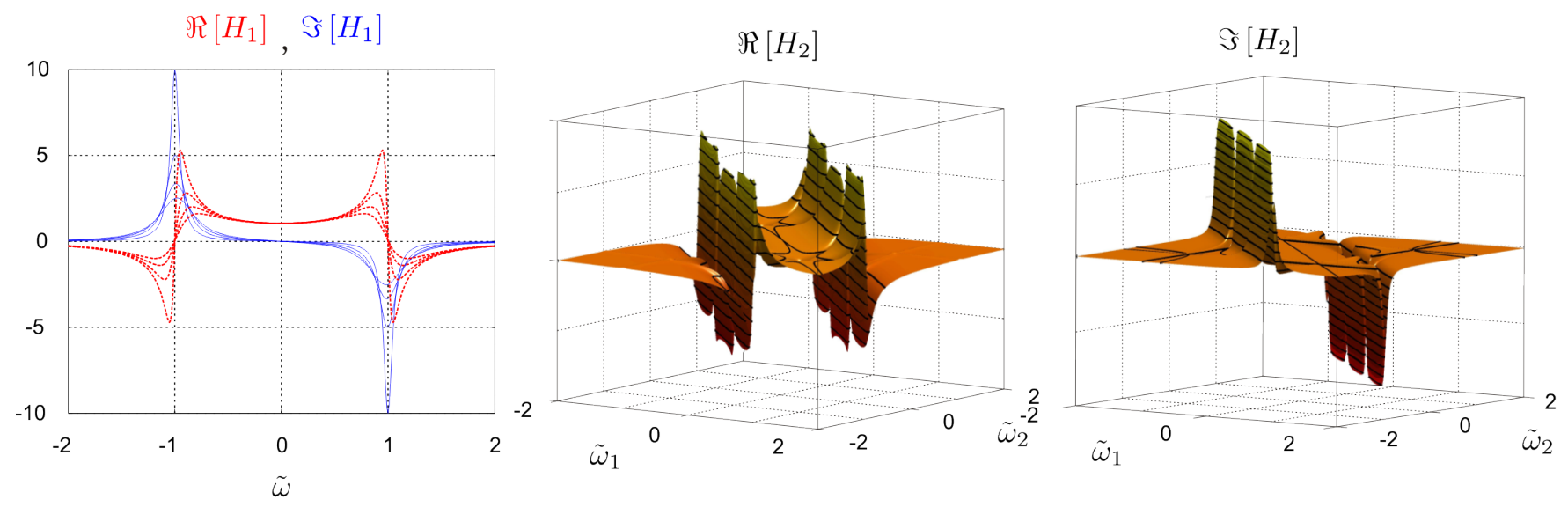

Fig. 1: Real and imaginary parts of the Volterra frequency response functions of the problem: $H_{1}(\tilde{\omega})$ is represented for $\xi=2.5 \%, 5 \%, 7.5 \%, 10 \%$ and $H_{2}\left(\tilde{\omega}_{1}, \tilde{\omega}_{2}\right)$ is represented for $\xi_{s}=\xi_{a}=2 \%$.

Observing that $H_{1}(\tilde{\omega})$ is at most of order $\xi^{-1}$, where $\tilde{\omega} \simeq 1, H_{2, u \dot{x}}$ might have the same order of magnitude as $H_{2, u^{2}}$, along the crests $\tilde{\omega}_{1} \simeq 1$ or $\tilde{\omega}_{2} \simeq 1$, and for $\operatorname{ord}\left(\xi_{a}\right)=\operatorname{ord}(\xi)$. This justifies our concern for not systematically discarding this parametric excitation term. Furthermore, it is readily seen that $H_{2, \dot{x}^{2}}$ might reach the same order of magnitude as $H_{2, u^{2}}$ too, but on a much smaller domain as it requires both $\tilde{\omega}_{1} \simeq 1$ and $\tilde{\omega}_{2} \simeq 1$. It is thus expected to be of secondary importance; this is confirmed next.

\subsection{Associated Linear Equations}

The Associated Linear Equations (ALE; Vazquez Feijoo et al. 2005) of a Volterra system are linear equations describing the dynamics of each term in the series expansion $(13)$. The ALE providing $\tilde{x}_{j}(\tilde{t})$ is forced by a nonlinear transformation involving the external excitation and/or the response at orders lower than $j$. For this reason, the ALEs can be integrated in a cascade manner without any closure issue. Besides, for some classes of nonlinear systems like the case at hand |Carassale and Kareem, 2014, the ALEs have a differential structure. In our case, they read (see Appendix A)

$$
\begin{aligned}
\tilde{x}_{0} & =\frac{1}{2 I_{u}} \\
\tilde{x}_{1}^{\prime \prime}+2\left(\xi_{s}+\xi_{a}\right) \tilde{x}_{1}^{\prime}+\tilde{x}_{1} & =\tilde{u} \\
\tilde{x}_{2}^{\prime \prime}+2\left(\xi_{s}+\xi_{a}\right) \tilde{x}_{2}^{\prime}+\tilde{x}_{2} & =I_{u}\left(\frac{\tilde{u}^{2}}{2}-2 \xi_{a} \tilde{u} \tilde{x}_{1}^{\prime}+2 \xi_{a}^{2} \tilde{x}_{1}^{\prime 2}\right) .
\end{aligned}
$$

At first glance, the numerical simulation of this set of ALEs might appear more involved than that of the nonlinear system (9), as it requires the solution of two differential equations instead of one. However, these two equations are linear and the commonly available numerical tools for the simulation of linear second order systems may be used, which makes its practical implementation rather straightforward and very efficient from a computational point of view.

These linear equations confirm that $\tilde{x}_{0}+\tilde{x}_{1}$ is the solution of the linear Gaussian problem (2), in its dimensionless form. Much more importantly, this ALE formulation provides a direct analysis of the orders of magnitude of the different components of the response. Beyond equation $\sqrt{19}$ showing that $\tilde{x}_{0}=\operatorname{ord}\left(I_{u}^{-1}\right)$ as seen also previously in (16), equation (20) indicates that $\tilde{x}_{1}$ is the solution of a linear system with unit timescale subjected to a unit variance low frequency turbulence, as $\alpha \ll 1$. It is therefore composed of an ord (1) background component and an $\operatorname{ord}\left(S_{\tilde{u}}(1) / \xi\right)$ resonant component, which might be leading depending on the relative smallness of $\xi$ and $S_{\tilde{u}}(1)$, this latter one being related to the smallness of $\alpha$. In wind engineering applications, the resonant component seldom exceeds the background one, so that $S_{\tilde{u}}(1) / \xi \sim 1$ in worst cases. Furthermore, the velocity response $\tilde{x}_{1}^{\prime}$ is typically assumed to be composed of the resonant component only so that $\tilde{x}_{1}^{\prime}=\operatorname{ord}\left(S_{\tilde{u}}(1) / \xi\right)$. The right-hand side of (21) indicates that $\tilde{x}_{2}$ scales with $I_{u}$ and is therefore expected to be small compared to $\tilde{x}_{0}$ and $\tilde{x}_{1}$, i.e. it marginally affects the variance of the response. Also, the orders of magnitude of the three terms in the right-hand side of (21), which are associated with the quadratic turbulence, the parametric and the quadratic velocity loading terms, are $1, S_{\tilde{u}}(1) \xi_{a} / \xi$ and $\left(S_{\tilde{u}}(1) \xi_{a} / \xi\right)^{2}$. Before solving the set of ALEs, we may thus claim that the third term, related to 
the quadratic velocity term, has small importance on the response, as it is one to two orders of magnitude smaller than its neighbors. The second term is also smaller than the first term, but acts along a different dynamics as $\tilde{u}^{2}$ is mainly low-frequency while $\tilde{u} \tilde{x}_{1}^{\prime}$ is mixed between the low-frequency turbulence and the resonance frequency.

In summary, this analysis confirms the well-known fact that the linear problem provides a very good estimation of the magnitude of the response, i.e. its variance. Consideration of the nonlinear problem thus concerns the proper estimation of the higher cumulants. It also indicates that the parametric excitation term $\tilde{u} \tilde{x}_{1}^{\prime}$ although smaller than the quadratic term $\tilde{u}^{2}$ might influence as much the higher statistics of the response, whilst the quadratic velocity feedback term $\tilde{x}_{1}^{\prime 2}$ is of secondary importance.

\subsection{Cumulants of the Response of the Second Order Model}

After this preliminary analysis on the different components of the response $\tilde{x}$, we now derive the expressions for the cumulants of the second order Volterra system, mainly for the second, third and fourth cumulants. They are approximations to the exacts cumulants $\kappa_{2}[\tilde{x}], \kappa_{3}[\tilde{x}], \kappa_{4}[\tilde{x}]$ of the solution $\tilde{x}$ of the nonlinear equation (9).

Because $\tilde{x} \simeq \tilde{x}_{0}+\tilde{x}_{1}+\tilde{x}_{2}$ in the second order Volterra model, the cumulants of $\tilde{x}$ might be obtained from those of its components, defined in (14). Application of standard theory of linear combinations of random variables Papoulis and Pillai, 2002 yields, after some algebra,

$$
\begin{aligned}
& \kappa_{2}[\tilde{x}] \simeq \kappa_{2}\left[\tilde{x}_{1}+\tilde{x}_{2}\right]=\kappa_{2}\left[\tilde{x}_{1}\right]+\kappa_{2}\left[\tilde{x}_{2}\right] \\
& \kappa_{3}[\tilde{x}] \simeq \kappa_{3}\left[\tilde{x}_{1}+\tilde{x}_{2}\right]=3 \kappa_{3}\left[\tilde{x}_{1}, \tilde{x}_{1}, \tilde{x}_{2}\right]+\kappa_{3}\left[\tilde{x}_{2}\right] \\
& \kappa_{4}[\tilde{x}] \simeq \kappa_{4}\left[\tilde{x}_{1}+\tilde{x}_{2}\right]=6 \kappa_{4}\left[\tilde{x}_{1}, \tilde{x}_{1}, \tilde{x}_{2}, \tilde{x}_{2}\right]+\kappa_{4}\left[\tilde{x}_{2}\right]
\end{aligned}
$$

where $\kappa_{j}[\cdot]$ represents, when used with a single argument, the $j^{\text {th }}$ (univariate) cumulant of the argument, while $\kappa_{j}[\cdot, \cdot, \ldots, \cdot]$ represents the $j^{t h}$ cross-cumulant associated with the product of the arguments. These equations show that the variance $\kappa_{2}$ and the third and fourth cumulants are obtained as the sum of two components each. Former studies Kareem, 1984 have shown that $\kappa_{2}\left[\tilde{x}_{1}\right]$ is the major contribution to the variance. The preliminary study here above confirms this trend as $\tilde{x}_{2} / \tilde{x}_{1}=$ ord $\left(I_{u}\right)$, leaving thus $\kappa_{2}\left[\tilde{x}_{2}\right]$ one to two orders of magnitude below $\kappa_{2}\left[\tilde{x}_{1}\right]$, at least for practical ranges of turbulence intensity. Following similar arguments, it is anticipated that $\kappa_{3}\left[\tilde{x}_{2}\right]$ and $\kappa_{4}\left[\tilde{x}_{2}\right]$ are negligible components of the third and fourth cumulants. In the following section, this is verified by integrating the ALEs and the full nonlinear system in a Monte Carlo simulation framework.

\subsection{Validation of the Second Order Model}

The accuracy of the second order Volterra model in approximating the nonlinearity of the problem is assessed by comparison of the cumulants of the response obtained with the full nonlinear equation and those obtained with the second order model. First, a realization of an input time series $\tilde{u}(\tilde{t})$, discretized with the constant time step $\Delta \tilde{t}$ and with length $\tilde{T}$ is generated by the spectral representation method (e.g. Di Paola 1998). Then, the nonlinear equation (11) is solved with an explicit Runge-Kutta $(2,3)$ solver, while the ALEs (20)-(21) are integrated adopting a linear approximation of the input within the time steps. The length of the time steps and the length of the simulated time series have been calibrated to incorporate all the significant spectral contributions in the system response. The fulfillment of this criterion for each point of the considered parameter space required the use of $\Delta \tilde{t}=0.1$ and $\tilde{T} \simeq 10^{5}$. The statistical moments of the response have been estimated through averaging the statistics calculated for 2000 simulations for each point in the parameter space. Both for the case of nonlinear equation and ALEs, the cumulants and cross-cumulants of $\tilde{x}$ (resp. $\tilde{x}_{1}$ and $\tilde{x}_{2}$ ) are computed from their statistical moments. These are obtained using an online averaging method, which avoids storage of long time series. Denoting the mathematical expectation operator by $E[\cdot]$, the cumulants of the response of the nonlinear system are obtained as

$$
\begin{aligned}
& \kappa_{2}[\tilde{x}]=E\left[\tilde{x}^{2}\right]-E[\tilde{x}]^{2} \\
& \kappa_{3}[\tilde{x}]=E\left[\tilde{x}^{3}\right]-3 E[\tilde{x}] E\left[\tilde{x}^{2}\right]+2 E[\tilde{x}]^{3} \\
& \kappa_{4}[\tilde{x}]=E\left[\tilde{x}^{4}\right]-4 E[\tilde{x}] E\left[\tilde{x}^{3}\right]+12 E[\tilde{x}]^{2} E\left[\tilde{x}^{2}\right]-3 E\left[\tilde{x}^{2}\right]^{2}-6 E[\tilde{x}]^{4}
\end{aligned}
$$

as per basic statistics Papoulis and Pillai, 2002. Similarly, in the second order Volterra model, the cumulants and cross-cumulants of the components $\tilde{x}_{1}$ and $\tilde{x}_{2}$ are obtained as

$$
\begin{aligned}
\kappa_{2}\left[\tilde{x}_{1}\right] & =E\left[\tilde{x}_{1}^{2}\right] \\
\kappa_{2}\left[\tilde{x}_{2}\right] & =E\left[\tilde{x}_{2}^{2}\right]-E\left[\tilde{x}_{2}\right]^{2}
\end{aligned}
$$



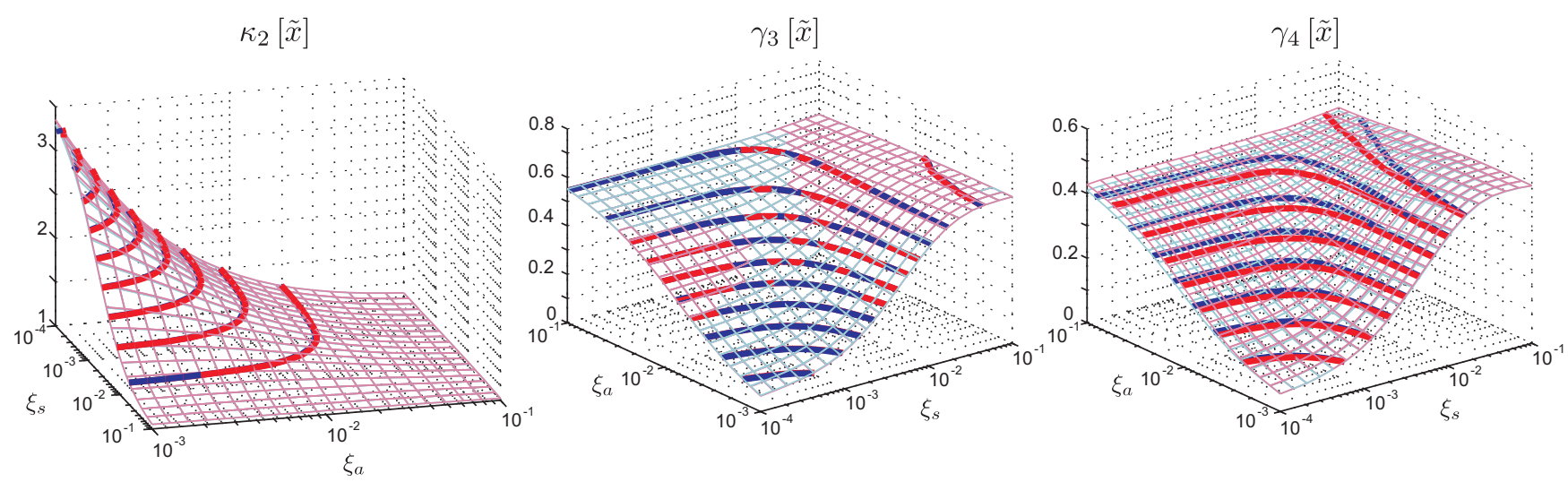

Fig. 2: Response of the nonlinear system (blue) and of the second order Volterra system (red). (a) Standard deviation, (b) Skewness coefficient, (c) Excess coefficient. Obtained for $I_{u}=0.2, \alpha=0.005$ and $S_{\tilde{u}}(\tilde{\omega} ; \alpha)$ given by (27). See online version for colors.

$$
\begin{aligned}
\kappa_{3}\left[\tilde{x}_{1}, \tilde{x}_{1}, \tilde{x}_{2}\right] & =E\left[\tilde{x}_{1}^{2} \tilde{x}_{2}\right]-E\left[\tilde{x}_{1}^{2}\right] E\left[\tilde{x}_{2}\right] \\
\kappa_{3}\left[\tilde{x}_{2}\right] & =E\left[\tilde{x}_{2}^{3}\right]-3 E\left[\tilde{x}_{2}\right] E\left[\tilde{x}_{2}^{2}\right]+2 E\left[\tilde{x}_{2}\right]^{3} \\
\kappa_{4}\left[\tilde{x}_{1}, \tilde{x}_{1}, \tilde{x}_{2}, \tilde{x}_{2}\right] & =E\left[\tilde{x}_{1}^{2} \tilde{x}_{2}^{2}\right]-E\left[\tilde{x}_{1}^{2}\right] E\left[\tilde{x}_{2}^{2}\right]-2 E\left[\tilde{x}_{1}^{2} \tilde{x}_{2}\right] E\left[\tilde{x}_{2}\right]+2 E\left[\tilde{x}_{1}^{2}\right] E\left[\tilde{x}_{2}\right]^{2} \\
\kappa_{4}\left[\tilde{x}_{2}\right] & =E\left[\tilde{x}_{2}^{4}\right]-4 E\left[\tilde{x}_{2}\right] E\left[\tilde{x}_{2}^{3}\right]+12 E\left[\tilde{x}_{2}\right]^{2} E\left[\tilde{x}_{2}^{2}\right]-3 E\left[\tilde{x}_{2}^{2}\right]^{2}-6 E\left[\tilde{x}_{2}\right]^{4}
\end{aligned}
$$

which is slightly simplified on account that $E\left[\tilde{x}_{1}\right]=E\left[\tilde{x}_{1} \tilde{x}_{2}\right]=0$, by construction of the Volterra series. The estimation of the cumulants of the response then follows from substitution of these expressions into (22)-(24).

Considering the dimensionless turbulence power spectral density

$$
S_{\tilde{u}}(\tilde{\omega} ; \alpha)=\frac{1}{\alpha} \frac{0.546}{\left(1+1.64\left|\frac{\tilde{\omega}}{\alpha}\right|\right)^{5 / 3}}
$$

which is the scaled version of $(3)$, considering also $I_{u}=0.2$ and $\alpha=0.005$, while varying the two remaining parameters $\xi_{s}$ and $\xi_{a}$, a parametric analysis was conducted. Figures 2 (a-c) show the standard deviation $\kappa_{2}[\tilde{x}]$, skewness coefficient $\gamma_{3}[\tilde{x}]=\kappa_{3}[\tilde{x}] / \kappa_{2}[\tilde{x}]^{3 / 2}$ and excess coefficient $\gamma_{4}[\tilde{x}]=\kappa_{4}[\tilde{x}] / \kappa_{2}[\tilde{x}]^{2}$ of the response, as a function of the structural and aerodynamic damping coefficients. It compares the results obtained for the nonlinear model (blue) and the second order Volterra model (red).

The response is mainly resonant in the region of small damping, for $\xi_{s} \lll 1$ and $\xi_{a} \lll 1$, where the standard deviation of the response grows unbounded (a more appropriate condition is $\xi \ll S_{\tilde{u}}(1)$ as shown next). In this region, the skewness and excess coefficients are small as a result of the extension of the memory lag of the linear underlying system and the central limit theorem. At the opposite, the response is mainly quasi-static for high dampings. There, the standard deviation of the response tends to 1 as a result of the scaling, while the skewness and excess coefficients tend to $3 I_{u}=0.6$ and $12 I_{u}^{2}=0.48$ respectively, which correspond to the cumulants of a quadratic transformation of a zero-mean $I_{u}$-standard deviation normal process.

The perfect agreement between the response of the nonlinear model and the Volterra model, for the second and third cumulants, demonstrates the suitability of the truncation to the second order, at least for the considered set of parameters. The agreement is acceptable for the fourth cumulant, and actually as good as $\xi_{a}$ is small as this parameter governs the nonlinearity in the model. A behind-the-scenes analysis has revealed that a third order Volterra model actually captures the fourth order response almost perfectly, for the same set of parameters again. We have however decided to limit the truncation to order 2, in order to allow for simple closed-form expressions in the sequel.

Varying the fixed parameters $I_{u}=0.2$ and $\alpha=0.005$ of this study might be the scope of an extensive exercise, but the ALEs suggest that an increase of the turbulence intensity $I_{u}$ would just amplify the skewness and excess coefficients, without affecting the quality of the agreement; similarly, a modification of the relative frequency of the turbulence $\alpha$ would displace the position of the inflexion points, while maintaining the agreement between both models.

We further take the opportunity of this validation stage to compare, in the second order Volterra model, the relative importance of the two contributions to the second, third and fourth cumulants respectively. To this purpose, 

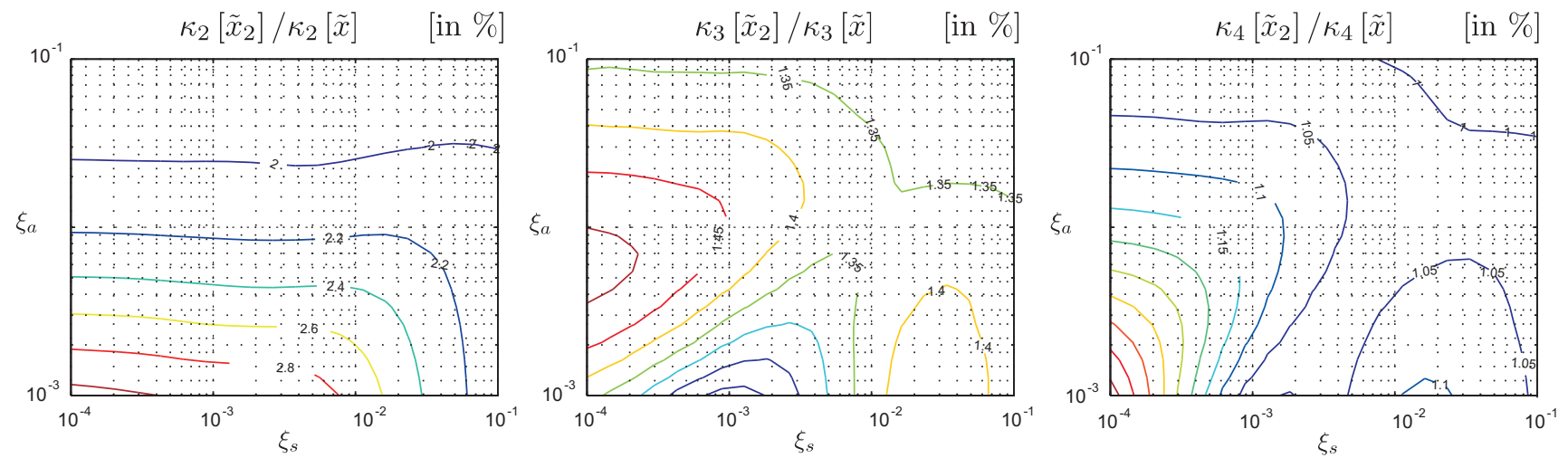

Fig. 3: Relative contribution of the component $\kappa_{j}\left[\tilde{x}_{2}\right]$ to the $j^{t h}$ cumulant of the response of the second order Volterra model. Obtained for $I_{u}=0.2, \alpha=0.005$ and $S_{\tilde{u}}(\tilde{\omega} ; \alpha)$ given by $(27)$. See online version for colors.

we represent the ratios $\kappa_{j}\left[\tilde{x}_{2}\right] / \kappa_{j}[\tilde{x}], j \in\{2,3,4\}$ in Fig. 3 for the same values of the problem parameters. The relative smallness $\tilde{x}_{2} \ll \tilde{x}_{1}$ is well confirmed in Fig. 3 a a and the contributions of $\kappa_{j}\left[\tilde{x}_{2}\right]$ to the total cumulants are seen to be less than $3 \%$ for the variance and less than $1 \%$ for the third and fourth cumulants. Based on these observations, we will neglect the term $\kappa_{j}\left[\tilde{x}_{2}\right]$ in the cumulants of the response, see $(22)-(24)$.

\section{Higher Order Spectra of the Second order Volterra Model}

Former works [Li et al. 1995 have established the expressions of the first four cumulants of the response of second and third order Volterra models. Nonetheless the application of the Multiple Timescale Spectral Analysis requires analytical expressions for the higher order spectra of the response. To the authors' best knowledge, these expressions have never been established before. They are developed in this Section, in the most general case of a second order Volterra model. The expressions given for the spectra of the response are not limited to the considered problem, but are general for any second order Volterra model characterized by the frequency repose functions $H_{1}(\omega)$ and $H_{2}\left(\omega_{1}, \omega_{2}\right)$.

\subsection{Second order response}

At the second order, the correlation function of the response $\tilde{x}(t)$ is given by

$$
R_{\tilde{x}}(\tau)=E[\tilde{x}(t) \tilde{x}(t+\tau)]
$$

with the fundamental property that the value at the origin corresponds to the second statistical moment. The substitution of $\tilde{x}(t)=\tilde{x}_{0}+\tilde{x}_{1}(t)+\tilde{x}_{2}(t)$ in this expression provides

$$
R_{\tilde{x}}(\tau)=\tilde{x}_{0}^{2}+2 \tilde{x}_{0} E\left[\tilde{x}_{2}(t)\right]+E\left[\tilde{x}_{1}(t) \tilde{x}_{1}(t+\tau)\right]+E\left[\tilde{x}_{2}(t) \tilde{x}_{2}(t+\tau)\right]
$$

on account that $E\left[\tilde{x}_{1}(t)\right]=E\left[\tilde{x}_{1}(t) \tilde{x}_{2}(t+\tau)\right]=E\left[\tilde{x}_{2}(t) \tilde{x}_{1}(t+\tau)\right]=0$ since they involve an odd power of $u$ after substitution of (14) for $\tilde{x}_{1}$ and $\tilde{x}_{2}$. The last two terms in (29) correspond to the correlation functions $R_{\tilde{x}_{1}}$ and $R_{\tilde{x}_{2}}$ of the components $\tilde{x}_{1}$ and $\tilde{x}_{2}$. Upon substitution of 14 for $\tilde{x}_{1}$ and $\tilde{x}_{2}$ and consideration that $\tilde{u}$ is a Gaussian process, they respectively read

$$
R_{\tilde{x}_{1}}(\tau)=\iint_{\mathbb{R}^{2}} h_{1}\left(\tau_{1}\right) h_{1}\left(\tau_{2}\right) R_{\tilde{u}}\left(\tau+\tau_{1}-\tau_{2}\right) d \tau_{1} d \tau_{2}
$$

and

$$
\begin{aligned}
R_{\tilde{x}_{2}}(\tau) & =\int \ldots \int_{\mathbb{R}^{4}} h_{2}\left(\tau_{1}, \tau_{2}\right) h_{2}\left(\tau_{3}, \tau_{4}\right) R_{\tilde{u}}\left(\tau_{1}-\tau_{2}\right) R_{\tilde{u}}\left(\tau_{3}-\tau_{4}\right) d \tau_{1} d \tau_{2} d \tau_{3} d \tau_{4} \\
& +\int \ldots \int_{\mathbb{R}^{4}} h_{2}\left(\tau_{1}, \tau_{2}\right) h_{2}\left(\tau_{3}, \tau_{4}\right) R_{\tilde{u}}\left(\tau+\tau_{1}-\tau_{3}\right) R_{\tilde{u}}\left(\tau+\tau_{2}-\tau_{4}\right) d \tau_{1} d \tau_{2} d \tau_{3} d \tau_{4}
\end{aligned}
$$




$$
+\int_{\mathbb{R}^{4}} \ldots \int_{2} h_{2}\left(\tau_{1}, \tau_{2}\right) h_{2}\left(\tau_{3}, \tau_{4}\right) R_{\tilde{u}}\left(\tau+\tau_{1}-\tau_{4}\right) R_{\tilde{u}}\left(\tau+\tau_{2}-\tau_{3}\right) d \tau_{1} d \tau_{2} d \tau_{3} d \tau_{4}
$$

where $R_{\tilde{u}}(\tau)=E[\tilde{u}(t) \tilde{u}(t+\tau)]$ is the covariance function of the (zero-mean) fluctuation velocity $\tilde{u}$. The side-byside Fourier transform of (29) and the substitution of the Volterra kernels $h_{1}$ and $h_{2}$ by the corresponding frequency response functions, see (15), provides the power spectral density of the response. Omitting the two Dirac terms centered on $\tilde{\omega}=0$ resulting from the first two terms in 29 and from the first term in (31) which does not depend on $\tau$, it reads

$$
S_{\tilde{x}}(\tilde{\omega})=S_{\tilde{x}_{1}}(\tilde{\omega})+S_{\tilde{x}_{2}}(\tilde{\omega})
$$

where

$$
\begin{aligned}
& S_{\tilde{x}_{1}}(\omega)=\left|H_{1}(\omega)\right|^{2} S_{\tilde{u}}(\omega), \\
& S_{\tilde{x}_{2}}(\omega)=2 \int_{\mathbb{R}}\left|H_{2}\left(\omega_{1}, \omega-\omega_{1}\right)\right|^{2} S_{\tilde{u}}\left(\omega_{1}\right) S_{\tilde{u}}\left(\omega-\omega_{1}\right) d \omega_{1} .
\end{aligned}
$$

Having discarded the Dirac terms related to the squared mean of the response, the power spectral density $S_{\tilde{x}}(\tilde{\omega})$ in (32) is therefore associated with the second cumulant of the response (variance), rather than its second raw moment (mean square). Accordingly, the integral of $(32)$ over the frequency domain $\omega \in \mathbb{R}$ returns the cumulant $\kappa_{2}[\tilde{x}]$ in $(22) ; \kappa_{2}\left[\tilde{x}_{1}\right]$ and $\kappa_{2}\left[\tilde{x}_{2}\right]$ are readily interpreted as the integrals of $S_{\tilde{x}_{1}}(\omega)$ and $S_{\tilde{x}_{2}}(\omega)$, respectively.

\subsection{Third order response}

With slightly more involved calculus, the bispectrum and the trispectrum of the response might be obtained in a very similar manner, but starting from the bicorrelation and tricorrelation functions. In particular, the bicorrelation function of the response is defined as

$$
R_{\tilde{x}}\left(\tau_{1}, \tau_{2}\right)=E\left[\tilde{x}(t) \tilde{x}\left(t+\tau_{1}\right) \tilde{x}\left(t+\tau_{2}\right)\right]
$$

with the fundamental property that the value at the origin corresponds to the third statistical moment. The function $R_{\tilde{x}}$ is used with two arguments to refer to the bicorrelation function. After some developments, following the same outline as above and especially neglecting the delta-Dirac terms, the cumulant-related bispectrum of the response is expressed as

$$
B_{\tilde{x}}\left(\tilde{\omega}_{1}, \tilde{\omega}_{2}\right)=B_{\tilde{x}_{112}}\left(\tilde{\omega}_{1}, \tilde{\omega}_{2}\right)+B_{\tilde{x}_{2}}\left(\tilde{\omega}_{1}, \tilde{\omega}_{2}\right)
$$

where

$$
\begin{aligned}
B_{\tilde{x}_{112}}\left(\tilde{\omega}_{1}, \tilde{\omega}_{2}\right) & =2 H_{1}\left(-\tilde{\omega}_{1}-\tilde{\omega}_{2}\right) H_{1}\left(\tilde{\omega}_{1}\right) H_{2}\left(\tilde{\omega}_{1}+\tilde{\omega}_{2},-\tilde{\omega}_{1}\right) S_{\tilde{u}}\left(\tilde{\omega}_{1}+\tilde{\omega}_{2}\right) S_{\tilde{u}}\left(\tilde{\omega}_{1}\right) \\
& +2 H_{1}\left(-\tilde{\omega}_{1}-\tilde{\omega}_{2}\right) H_{1}\left(\tilde{\omega}_{2}\right) H_{2}\left(\tilde{\omega}_{1}+\tilde{\omega}_{2},-\tilde{\omega}_{2}\right) S_{\tilde{u}}\left(\tilde{\omega}_{1}+\tilde{\omega}_{2}\right) S_{\tilde{u}}\left(\tilde{\omega}_{2}\right) \\
& +2 H_{1}\left(\tilde{\omega}_{1}\right) H_{1}\left(\tilde{\omega}_{2}\right) H_{2}\left(-\tilde{\omega}_{1},-\tilde{\omega}_{2}\right) S_{\tilde{u}}\left(\tilde{\omega}_{1}\right) S_{\tilde{u}}\left(\tilde{\omega}_{2}\right)
\end{aligned}
$$

and

$$
\begin{array}{r}
B_{\tilde{x}_{2}}\left(\tilde{\omega}_{1}, \tilde{\omega}_{2}\right)=8 \int_{\mathbb{R}} H_{2}\left(-\tilde{\omega}_{1}-\tilde{\omega}_{3},-\tilde{\omega}_{2}+\tilde{\omega}_{3}\right) H_{2}\left(\tilde{\omega}_{1}+\tilde{\omega}_{3},-\tilde{\omega}_{3}\right) \\
H_{2}\left(\tilde{\omega}_{2}-\tilde{\omega}_{3}, \tilde{\omega}_{3}\right) S_{\tilde{u}}\left(\tilde{\omega}_{1}+\tilde{\omega}_{3}\right) S_{\tilde{u}}\left(\tilde{\omega}_{2}-\tilde{\omega}_{3}\right) S_{\tilde{u}}\left(\tilde{\omega}_{3}\right) d \tilde{\omega}_{3} .
\end{array}
$$

The integration of this bispectrum over the frequency domain $\left(\tilde{\omega}_{1}, \tilde{\omega}_{2}\right) \in \mathbb{R}^{2}$ provides the third cumulant of the response $\kappa_{3}[\tilde{x}]$, as defined in $(23)$. Again, a stepwise development of this expression — which is anyway confirmed by the composition of the factors in $B_{\tilde{x}_{112}}$ and $B_{\tilde{x}_{2}}$ - would indicate that the two components $B_{\tilde{x}_{112}}$ and $B_{\tilde{x}_{2}}$ are actually associated with the contributions $3 \kappa_{3}\left[\tilde{x}_{1}, \tilde{x}_{1}, \tilde{x}_{2}\right]$ and $\kappa_{3}\left[\tilde{x}_{2}\right]$ to the total cumulant. Since the second term has been shown to be smaller, only the component $B_{\tilde{x}_{112}}$ will be considered next. 


\subsection{Fourth order response}

At the fourth order, the total trispectrum of the response is expressed as

$$
R_{\tilde{x}}\left(\tau_{1}, \tau_{2}, \tau_{3}\right)=E\left[\tilde{x}(t) \tilde{x}\left(t+\tau_{1}\right) \tilde{x}\left(t+\tau_{2}\right) \tilde{x}\left(t+\tau_{3}\right)\right]
$$

On substituting $\tilde{x}(t)=x_{0}+\tilde{x}_{1}(t)+\tilde{x}_{2}(t)$ in this expression, together with the definitions of $\tilde{x}_{1}(t)$ and $\tilde{x}_{2}(t)$, converting to the threefold frequency domain and finally discarding delta-Dirac terms that do not contribute to the cumulant of the response, we obtain the general expression of the trispectrum as

$$
T_{\tilde{x}}\left(\tilde{\omega}_{1}, \tilde{\omega}_{2}, \tilde{\omega}_{3}\right)=T_{\tilde{x}_{1122}}\left(\tilde{\omega}_{1}, \tilde{\omega}_{2}, \tilde{\omega}_{3}\right)+T_{\tilde{x}_{2}}\left(\tilde{\omega}_{1}, \tilde{\omega}_{2}, \tilde{\omega}_{3}\right)
$$

with

$$
\begin{aligned}
& T_{\tilde{x}_{1122}}\left(\tilde{\omega}_{1}, \tilde{\omega}_{2}, \tilde{\omega}_{3}\right)=4 \sum_{\alpha, \beta, \gamma \in\{1,2,3\}} \\
& \alpha \neq \beta \neq \gamma \\
& H_{1}\left(\tilde{\omega}_{\alpha}\right) S_{\tilde{u}}\left(\tilde{\omega}_{\alpha}\right)\left[H_{1}\left(-\tilde{\omega}_{\alpha}-\tilde{\omega}_{\beta}-\tilde{\omega}_{\gamma}\right) H_{2}\left(-\tilde{\omega}_{\alpha}, \tilde{\omega}_{\alpha}+\tilde{\omega}_{\gamma}\right)\right. \\
& H_{2}\left(\tilde{\omega}_{\alpha}+\tilde{\omega}_{\beta}+\tilde{\omega}_{\gamma},-\tilde{\omega}_{\alpha}-\tilde{\omega}_{\gamma}\right) S_{\tilde{u}}\left(\tilde{\omega}_{\alpha}+\tilde{\omega}_{\beta}+\tilde{\omega}_{\gamma}\right) S_{\tilde{u}}\left(\tilde{\omega}_{\alpha}+\tilde{\omega}_{\gamma}\right) \\
& \left.+H_{1}\left(\tilde{\omega}_{\beta}\right) H_{2}\left(-\tilde{\omega}_{\alpha},-\tilde{\omega}_{\beta}-\tilde{\omega}_{\gamma}\right) H_{2}\left(\tilde{\omega}_{\beta}+\tilde{\omega}_{\gamma},-\tilde{\omega}_{\beta}\right) S_{\tilde{u}}\left(\tilde{\omega}_{\beta}\right) S_{\tilde{u}}\left(\tilde{\omega}_{\beta}+\omega_{1}, 0\right)\right]
\end{aligned}
$$

where the summation is performed on all six possible permutations of the indexes $\alpha, \beta$, $\gamma$ in the set $\{1,2,3\}$. The (somewhat longer) expression of $T_{\tilde{x}_{2}}\left(\tilde{\omega}_{1}, \tilde{\omega}_{2}, \tilde{\omega}_{3}\right)$ is an integral over $\mathbb{R}$ of a product of four factors in $H_{2}$ and three factors in $S_{\tilde{u}}$, similarly to (33) and (37). It is again possible to show that $T_{\tilde{x}_{2}}$ is associated with the small contribution $\kappa_{4}\left[\tilde{x}_{2}\right]$ to the fourth cumulant of the response, while the leading term, $6 \kappa_{4}\left[\tilde{x}_{1}, \tilde{x}_{1}, \tilde{x}_{2}, \tilde{x}_{2}\right]$ corresponds to the integral of $T_{\tilde{x}_{1122}}$ over the frequency space. The expression of $T_{\tilde{x}_{2}}$ is therefore not given here for conciseness as it will be neglected anyway in the sequel.

\section{Cumulants of the Response under Low-Frequency Turbulence}

The cumulants of the response are determined by integration of the corresponding spectra over the frequency space. The analytical computation of these integrals is difficult, if not impossible, for most common expressions of the power spectral density of wind turbulence. However, these integrals may be considerably simplified by considering the existence of various timescales in the problem, which consequently drops by one the order of integration. For instance, in the seminal work of Davenport [1961] related to the background/resonant decomposition in the context of single degree-of-freedom systems subject to low frequency loadings, the variance of the response is obtained without having recourse to any integration of the spectral density, i.e. no integration instead of integration in a 1-D space, formally. The idea was extended to similar higher-order linear applications [Denoël, 2009, Denoël, 2011] and recently generalized to multi-degree-of-freedom and nonlinear systems [Denoël, 2015]. This technique identifies the different contributions to the integral, then sequentially focuses on each of them with a game of stretch-and-shrink operations. In this Section, this Multiple Timescale Spectral Analysis is applied to the determination of the first four cumulants of the response of the second order Volterra model. In each case, the developments are based on the distinctness of the peaks in the response, that result from the small parameter $\alpha$ separating the fast dynamics of the structural resonance and the low frequency excitation of the wind turbulence.

\subsection{Second order response}

The variance of the response $\kappa_{2}[\tilde{x}]$ is obtained by integration of the power spectral density $S_{\tilde{x}}(\tilde{\omega})$. In the second order Volterra model, it results from the integration of two terms, among which only $S_{\tilde{x}_{1}}(\tilde{\omega})$ is considered. This term actually corresponds to the well-known first order response $x_{1}(t)$ of a linear oscillator. It exhibits one background peak in the vicinity of the origin $\tilde{\omega} \simeq 0$ with a halfheight width of order $\alpha$ and two symmetrical resonance peaks located at $\tilde{\omega}= \pm 1$ with a halfheight width of order $\xi$. The application of a classical decomposition Davenport, 1961] yields background and resonant components which are respectively given by

$$
\begin{aligned}
\kappa_{2, b}\left[\tilde{x}_{1}\right] & =\int_{\mathbb{R}}\left|H_{1}(0)\right|^{2} S_{\tilde{u}}(\tilde{\omega}) \mathrm{d} \tilde{\omega}=1, \\
\kappa_{2, r}\left[\tilde{x}_{1}\right] & =\frac{\pi S_{\tilde{u}}(1)}{2 \xi}
\end{aligned}
$$



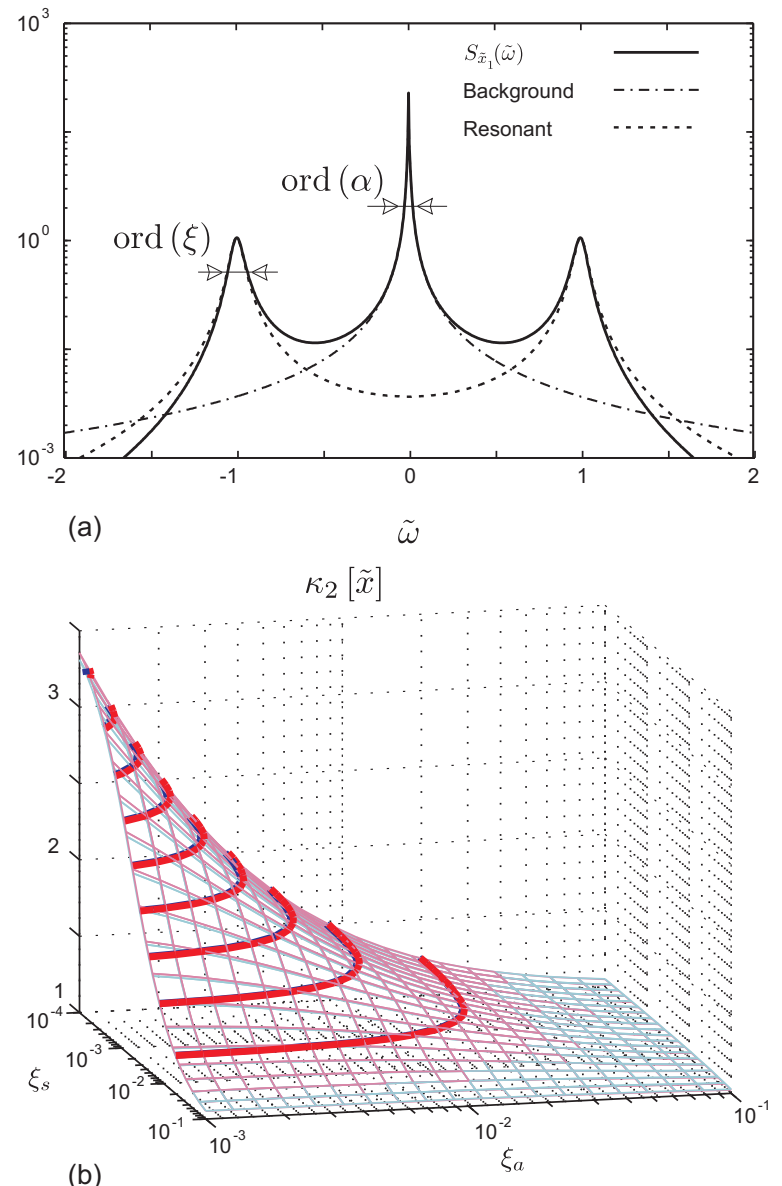

Fig. 4: (a) Power spectral density of the response $S_{\tilde{x}_{1}}(\tilde{\omega})$ and its components, obtained for $\xi_{s}=\xi_{a}=0.02, \alpha=0.005$ and $S_{\tilde{u}}(\tilde{\omega} ; \alpha)$ given by $(27)$; (b) Comparison of the variance of the response obtained with the analytical approximation (blue) and with the second Volterra model (red); $I_{u}=0.2, \alpha=0.005$. 
so that the variance of the linear response reads

$$
\kappa_{2}[\tilde{x}] \simeq \kappa_{2}\left[\tilde{x}_{1}\right]=1+\beta
$$

where

$$
\beta:=\frac{\kappa_{2, r}\left[\tilde{x}_{1}\right]}{\kappa_{2, b}\left[\tilde{x}_{1}\right]}
$$

is defined as the resonant-to-background ratio.

The formal application of the Multiple Timescale Spectral Analysis recovers this result [Denoël, 2011]. This is briefly summarized here as a prelude to the higher order responses, which are treated in a similar fashion in the sequel. Subtracting off the trivial background component from the integral of $S_{\tilde{x}_{1}}$, we are left with the remainder

$$
\rho_{2}=\int_{\mathbb{R}}\left[\left|H_{1}(\tilde{\omega})\right|^{2}-1\right] S_{\tilde{u}}(\tilde{\omega}) \mathrm{d} \tilde{\omega}
$$

which is mainly composed of two resonance peaks located at $\tilde{\omega}= \pm 1$. We focus on the rightmost peak with the coordinate stretching $\tilde{\omega}=1+\left(\xi_{s}+\xi_{a}\right) \eta_{1}$ and the Jacobian $\mathrm{d} \tilde{\omega}=\xi \mathrm{d} \eta_{1}$; the factor into the brackets becomes

$$
\left|H_{1}\left(\tilde{\omega}\left(\eta_{1}\right)\right)\right|^{2}-1=\frac{\left(1+\eta_{1} \xi\right)^{2}\left(1-4 \xi^{2}-2 \eta_{1} \xi-\eta_{1}^{2} \xi^{2}\right)}{\xi^{2}\left(4+8 \eta_{1} \xi+4 \eta_{1}^{2}\left(1+\xi^{2}\right)+4 \eta_{1}^{3} \xi+\eta_{1}^{4} \xi^{2}\right)}
$$

but is drastically simplified on account that $\xi \ll 1$ and that $\eta_{1}=\operatorname{ord}(1)$ on the interval of interest. Indeed, many terms in the above expression drop and the local approximation is

$$
\left|H_{1}\left(\tilde{\omega}\left(\eta_{1}\right)\right)\right|^{2}-1 \simeq \frac{1}{\xi^{2}\left(4+4 \eta_{1}^{2}\right)},
$$

which has just one pole in the region $\Im>0$ of the complex plane, i.e. one peak on the real axis, instead of two in (46). Further assuming $S_{\tilde{u}}\left(1+\xi \eta_{1}\right) \simeq S_{\tilde{u}}(1)$ for $\eta_{1}=\operatorname{ord}(1)$, and multiplying the result by two in order to account for both peaks, we recover well 42 ,

$$
\kappa_{2, r}\left[\tilde{x}_{1}\right]=2 \int_{\mathbb{R}} \frac{1}{\xi^{2}\left(4+4 \eta_{1}^{2}\right)} S_{\tilde{u}}(1) \xi \mathrm{d} \eta_{1}=\frac{\pi S_{\tilde{u}}(1)}{2 \xi}=\beta .
$$

The normalization of the power spectral density of the velocity fluctuation is such that $S_{\tilde{u}}(1)$ is also a small number of the problem. Indeed, because of the normalization property (6), because of the globally decreasing pattern of $S_{\tilde{u}}$ and because $S_{\tilde{u}}(0)=\operatorname{ord}\left(\alpha^{-1}\right)$, it is possible to assert that $S_{\tilde{u}}(1) \lesssim \alpha$, i.e. smaller than or at most of the same order of magnitude as $\alpha$, without the possibility to be more specific in the general case. Whether the resonant contribution $\kappa_{2, r}$ is larger or not than the background one $\kappa_{2, b}$ is a matter of relative smallnesses of $S_{\tilde{u}}(1)$ and $\xi=\xi_{s}+\xi_{a}$. The parameter $\beta$ measures this ratio and indicates the type of vibrations, oscillatory or quasi-static.

Figure $4 \mathrm{~b}$ compares the standard deviation resulting from this analytical analysis (in blue) and that resulting from the Monte Carlo analysis of the response of the second order Volterra model (in red). The difference between the two results is barely noticeable; nothing amazing as this background/resonant decomposition is known to be efficient (a result of the timescale separation) and since the contribution of nonlinear terms to the variance of the response is small.

\subsection{Third order response}

The third cumulant of the response is obtained by integration of the bispectrum given in (35), which is composed of two contributions, in the second order Volterra model. Only the first contribution $B_{\tilde{x}_{112}}\left(\tilde{\omega}_{1}, \tilde{\omega}_{2}\right)$ is studied here as it constitutes the most important part in the cumulant, i.e. $3 \kappa_{3}\left[\tilde{x}_{1}, \tilde{x}_{1}, \tilde{x}_{2}\right]$. This contribution is itself composed of three terms, see (36), that contribute equally to the cumulant for symmetry reasons. We will thus focus on only one term, namely

$$
b_{\tilde{x}_{112}}\left(\tilde{\omega}_{1}, \tilde{\omega}_{2}\right)=2 H_{1}\left(\tilde{\omega}_{1}\right) H_{1}\left(\tilde{\omega}_{2}\right) H_{2}\left(-\tilde{\omega}_{1},-\tilde{\omega}_{2}\right) S_{\tilde{u}}\left(\tilde{\omega}_{1}\right) S_{\tilde{u}}\left(\tilde{\omega}_{2}\right)
$$

then multiply the result by 3 in order to obtain the cumulant of the response. This function is represented in Fig. 5-a. It exhibits a quasi-static peak at $\left(\tilde{\omega}_{1}, \tilde{\omega}_{2}\right)=(0,0)$ and four biresonance peaks of equal height as discussed below. They are approximated with the Multiple Timescale Spectral Analysis method. 


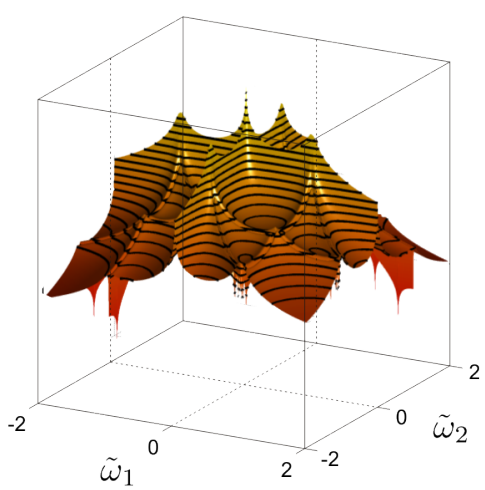

(a)

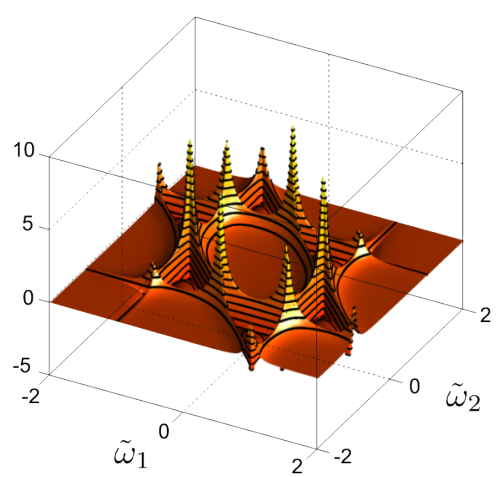

(b)

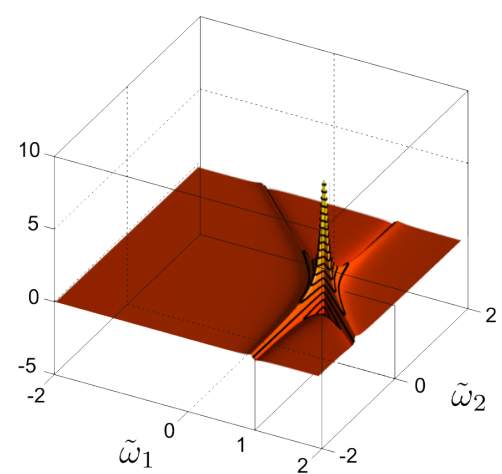

(c)

Fig. 5: (a) The first term $b_{\tilde{x}_{112}}$ of the bispectrum of the response $B_{\tilde{x}_{112}}\left(\tilde{\omega}_{1}, \tilde{\omega}_{2}\right)$ features one resonant peak and four biresonant peaks, (b) kernel-related factors in $b_{\tilde{x}_{112}}$ featuring six biresonance high peaks and six low peaks, (c) local approximation of the kernel-related factors in the neighborhood of $\left(\tilde{\omega}_{1}, \tilde{\omega}_{2}\right)=(1,0)$.

Numerical values: $\alpha=0.005, \xi=\xi_{a}=0.02, I_{u}=0.1$ and $S_{\tilde{u}}(\tilde{\omega} ; \alpha)$ given by (27).

The background component is readily obtained by integrating $b_{\tilde{x}_{112}}$ in the very low frequency range and multiplying by 3 to account for the three terms in the bispectrum, i.e.

$$
\kappa_{3, b}\left[\tilde{x}_{1}, \tilde{x}_{1}, \tilde{x}_{2}\right]=3\left(2 H_{1}(0) H_{1}(0) H_{2}(-0,-0) \iint_{\mathbb{R}^{2}} S_{\tilde{u}}\left(\tilde{\omega}_{1}\right) S_{\tilde{u}}\left(\tilde{\omega}_{2}\right) \mathrm{d} \tilde{\omega}_{1} \mathrm{~d} \tilde{\omega}_{2}\right)=3 I_{u} .
$$

With no approximation, the remainder in the integration of $b_{\tilde{x}_{112}}$ is expressed as

$$
\rho_{3}=\iint_{\mathbb{R}^{2}}\left[2 H_{1}\left(\tilde{\omega}_{1}\right) H_{1}\left(\tilde{\omega}_{2}\right) H_{2}\left(-\tilde{\omega}_{1},-\tilde{\omega}_{2}\right)-I_{u}\right] S_{\tilde{u}}\left(\tilde{\omega}_{1}\right) S_{\tilde{u}}\left(\tilde{\omega}_{2}\right) \mathrm{d} \tilde{\omega}_{1} \mathrm{~d} \tilde{\omega}_{2} .
$$

The factor into the brackets has the symmetry properties of a bispectrum and features thus a multiple of six peaks, see Fig. 5-b. Among them, the high biresonance peaks are located at $\left(\omega_{1}, \omega_{2}\right)=( \pm 1,0),(0, \pm 1)$ and $\pm(1,-1)$. These latter two peaks are however canceled out after multiplication by $S_{\tilde{u}}\left(\tilde{\omega}_{1}\right) S_{\tilde{u}}\left(\tilde{\omega}_{2}\right)$ which rapidly decreases as $\omega_{1}$ and $\omega_{2}$ depart from the planes $\omega_{1}=0$ and $\omega_{2}=0$. As a consequence, the remainder $\rho_{3}$ is essentially composed of four biresonance peaks, as those illustrated in Fig. 5 a. For symmetry reasons again, these four peaks contribute equally. We only focus on the peak located in $\left(\tilde{\omega}_{1}, \tilde{\omega}_{2}\right)=(1,0)$ then multiply the subsequent result by 4 . To do so, the stretched coordinates

$$
\tilde{\omega}_{1}=1+\left(\xi_{s}+\xi_{a}\right) \eta_{1} \quad ; \quad \tilde{\omega}_{2}=\left(\xi_{s}+\xi_{a}\right) \eta_{2}
$$

are introduced, with the Jacobian $|J|=\left(\xi_{s}+\xi_{a}\right)^{2}:=\xi^{2}$. This stretching aims at identifying in (51) the local contribution spanning a disk of unit radius in the $\left(\eta_{1}, \eta_{2}\right)$ coordinates. A local approximation of the integrand is therefore obtained by considering the smallness of $\xi_{s}$ and $\xi_{a}$ and considering that $\eta_{1}$ and $\eta_{2}$ are of order 1 , at most. After some developments, the factor in the brackets is simplified and we obtain

$$
\rho_{3}=\iint_{\mathbb{R}^{2}} I_{u} \frac{\left(\mathrm{i}+\eta_{1}\right) \xi-\mathrm{i} \xi_{a}}{4\left(1+\eta_{1}^{2}\right)\left(\mathrm{i}+\eta_{1}+\eta_{2}\right) \xi^{3}} S_{\tilde{u}}\left[\tilde{\omega}_{1}\left(\eta_{1}\right)\right] S_{\tilde{u}}\left[\tilde{\omega}_{2}\left(\eta_{2}\right)\right] \xi^{2} \mathrm{~d} \eta_{1} \mathrm{~d} \eta_{2}
$$

where the fraction is well integrable in the far field and presents a single peak in $\left(\eta_{1}, \eta_{2}\right)=(0,0)$ instead of the multiple peaks in the product $H_{1}\left(\tilde{\omega}_{1}\right) H_{1}\left(\tilde{\omega}_{2}\right) H_{2}\left(-\tilde{\omega}_{1},-\tilde{\omega}_{2}\right)$. This is thus an appropriate local approximation, in the sense of the Multiple Timescale Spectral Approach [Denoël 2015] and is represented in Fig. 5.c, which indeed confirms the extraction of one peak out of those represented in Fig. 5 -b.

Assuming $S_{\tilde{u}}\left(\tilde{\omega}_{1}\left(\eta_{1}\right)\right) \simeq S_{\tilde{u}}(1)$ over the unit disk authorizes a closed-form integration along $\eta_{1}$. On keeping only the real part, the one of interest, the remainder simplifies into

$$
\rho_{3}=I_{u} S_{\tilde{u}}(1) \iint_{\mathbb{R}^{2}} \frac{\left(1+\eta_{1}^{2}+\eta_{1} \eta_{2}\right) \xi-\xi_{a}}{4\left(1+\eta_{1}^{2}\right)\left(1+\left(\eta_{1}+\eta_{2}\right)^{2}\right) \xi^{3}} S_{\tilde{u}}\left[\tilde{\omega}_{2}\left(\eta_{2}\right)\right] \xi^{2} \mathrm{~d} \eta_{1} \mathrm{~d} \eta_{2}
$$




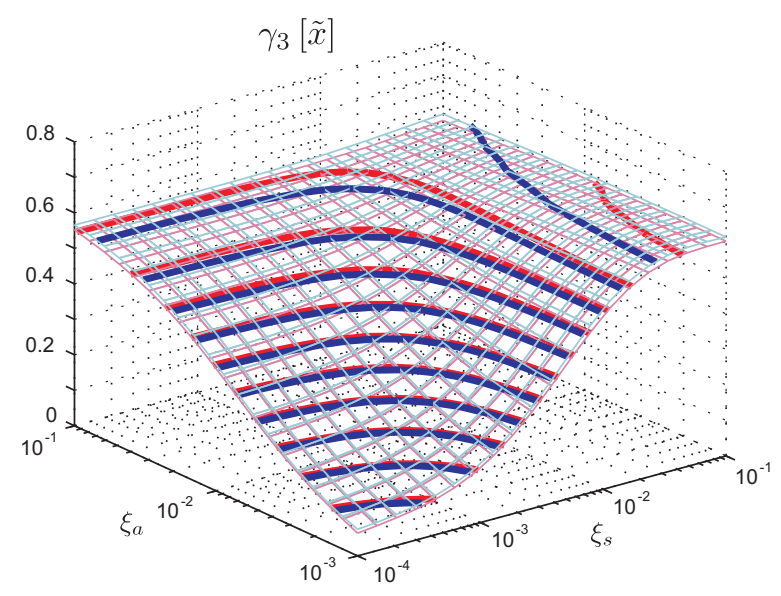

Fig. 6: Comparison of the skewness coefficient of the response obtained with the analytical approximation (blue) and with the second Volterra model (red) $I_{u}=0.2, \alpha=0.005$ and $S_{\tilde{u}}(\tilde{\omega} ; \alpha)$ given by (27).

$$
=I_{u} S_{\tilde{u}}(1) \frac{\pi}{2} \frac{2 \xi_{s}+\xi_{a}}{\xi_{s}+\xi_{a}} \int_{\mathbb{R}} \frac{S_{\tilde{u}}\left[\tilde{\omega}_{2}\left(\eta_{2}\right)\right]}{4+\eta_{2}^{2}} \mathrm{~d} \eta_{2}
$$

Multiplying this expression by 12 to take into consideration the factors 3 and 4 left behind earlier, and reverting back to the original frequency space, the total biresonant component reads

$$
\kappa_{3, r}\left[\tilde{x}_{1}, \tilde{x}_{1}, \tilde{x}_{2}\right]=6 \pi I_{u} S_{\tilde{u}}(1)\left(2 \xi_{s}+\xi_{a}\right) \int_{\mathbb{R}} \frac{S_{\tilde{u}}(\tilde{\omega})}{\tilde{\omega}^{2}+4\left(\xi_{s}+\xi_{a}\right)^{2}} \mathrm{~d} \tilde{\omega} .
$$

The total response, approximated as the sum of the background (50) and the biresonant (55) components, finally reads

$$
\kappa_{3}\left[\tilde{x}_{1}, \tilde{x}_{1}, \tilde{x}_{2}\right]=3 I_{u}+3 \pi I_{u} \frac{S_{\tilde{u}}(1)}{\xi}\left(1-\frac{\xi_{a}}{2 \xi}\right) \mathcal{I}_{1}\left(\xi_{s}+\xi_{a} ; S_{\tilde{u}}\right)
$$

where the integral

$$
\mathcal{I}_{1}\left(\xi ; S_{\tilde{u}}(\tilde{\omega} ; \alpha)\right)=4 \xi^{2} \int_{\mathbb{R}} \frac{S_{\tilde{u}}(\tilde{\omega} ; \alpha)}{\tilde{\omega}^{2}+4 \xi^{2}} \mathrm{~d} \tilde{\omega}
$$

represents the variance of a lowpass-filtered version of $\tilde{u}(\tilde{t})$. It is essentially smaller than unity and depends on the relative smallness of $\xi$ and $\alpha$; for $\xi \gg \alpha, \mathcal{I}_{1} \simeq 1$; for $\xi \ll \alpha, \mathcal{I}_{1} \simeq 2 \pi \xi S_{\tilde{u}}(0 ; \alpha) \ll 1$. Therefore, whether the background or the biresonant component of the response leads in the third cumulant is not only a matter of the smallness of $S_{\tilde{u}}(1) / \xi$ as for the second order response; it is also contingent upon the smallness of integral $\mathcal{I}_{1}$ through the ratio $\xi / \alpha$.

The skewness coefficient is usually preferred over the third cumulant, for ease of interpretability reasons. With the multiple timescale approximation at second and third orders, and limiting the computation of the statistics to the leading components $\kappa_{2}\left[\tilde{x}_{1}\right]$ and $\kappa_{3}\left[\tilde{x}_{1}, \tilde{x}_{1}, \tilde{x}_{2}\right]$ as above, we obtain

$$
\gamma_{3}[\tilde{x}]=3 I_{u} \frac{1+2 \beta\left(1-\frac{\xi_{a}}{2 \xi}\right) \mathcal{I}_{1}(\xi)}{(1+\beta)^{3 / 2}} .
$$

For the same set of parameters as the above illustrations, Figure 6 shows the skewness coefficient obtained with this relation (in blue) and with the Monte Carlo simulation of the second order Volterra system (in red). The very good agreement demonstrates the quality of the analytical approximation. It is as accurate as $\xi$ and $\alpha$ are small, since this favors the timescale separation.

\subsection{Fourth order response}

The fourth cumulant of the response is obtained by integration of the trispectrum, which is also composed of two terms in the second order Volterra model. Since it brings the major contribution to the fourth cumulant, see Section 


\begin{tabular}{cccc} 
B & BR-(I) & BR-(II) & R \\
\hline \hline$(0,0,0)$ & $(-1,0,1)$ & $(0,-1,1)$ & $(-1,-1,1)$ \\
& $(0,-1,0)$ & $(0,1,-1)$ & $(-1,1,1)$ \\
& $(0,1,0)$ & & $(1,-1,-1)$ \\
& $(1,0,-1)$ & & $(1,1,-1)$
\end{tabular}

Tab. 1: List of the locations $\left(\tilde{\omega}_{1}, \tilde{\omega}_{2}, \tilde{\omega}_{3}\right)$ and types of the main peaks in the trispectrum. B stands for background, $\mathrm{R}$ for triresonant and BR for mixed background/resonant.

3, only the term $T_{\tilde{x}_{1122}}\left(\tilde{\omega}_{1}, \tilde{\omega}_{2}, \tilde{\omega}_{3}\right)$ is considered next. This term is a sum of twelve terms, see (40), that contribute equally to the cumulant for symmetry reasons. We will thus focus on only one term, namely

$$
\begin{gathered}
t_{\tilde{x}}\left(\tilde{\omega}_{1}, \tilde{\omega}_{2}, \tilde{\omega}_{3}\right)=4 H_{1}\left(-\tilde{\omega}_{1}-\tilde{\omega}_{2}-\tilde{\omega}_{3}\right) H_{1}\left(\tilde{\omega}_{1}\right) H_{2}\left(-\tilde{\omega}_{1}, \tilde{\omega}_{1}+\tilde{\omega}_{3}\right) H_{2}\left(\tilde{\omega}_{1}+\tilde{\omega}_{2}+\tilde{\omega}_{3},-\tilde{\omega}_{1}-\tilde{\omega}_{3}\right) \\
S_{\tilde{u}}\left(\tilde{\omega}_{1}+\tilde{\omega}_{2}+\tilde{\omega}_{3}\right) S_{\tilde{u}}\left(\tilde{\omega}_{1}\right) S_{\tilde{u}}\left(\tilde{\omega}_{1}+\tilde{\omega}_{3}\right),
\end{gathered}
$$

then multiply the results by 12 in order to obtain the total cumulant of the response. It becomes tedious to proceed to a graphical representation of this function and to identify the regions of the frequency space which contribute the most to the total cumulant. Instead, we perform a systematic scanning of the regions where $t_{\tilde{x}}\left(\tilde{\omega}_{1}, \tilde{\omega}_{2}, \tilde{\omega}_{3}\right)$ might possibly be large, namely under the conditions $\tilde{\omega} \sim 0$ for $S_{\tilde{u}}(\tilde{\omega}), \tilde{\omega}= \pm 1$ for $H_{1}(\tilde{\omega})$ and $\tilde{\omega}_{1}+\tilde{\omega}_{2}= \pm 1$ for $H_{2}\left(\tilde{\omega}_{1}, \tilde{\omega}_{2}\right)$. The possible peaks in $t_{\tilde{x}}$ might be identified by fixing the triplet $\left(\tilde{\omega}_{1}, \tilde{\omega}_{2}, \tilde{\omega}_{3}\right)$ to values that maximize at least three of its seven factors. Combinatory tells us that this could be achieved in 35 different ways that could however be grouped in four groups $(35=1+12+18+4)$. In the first group (B), the three selected factors are those involving $S_{\tilde{u}}$. The values of $\tilde{\omega}_{1}, \tilde{\omega}_{2}$ and $\tilde{\omega}_{3}$ are thus determined by setting that the arguments $\tilde{\omega}_{1}+\tilde{\omega}_{2}+\tilde{\omega}_{3}, \tilde{\omega}_{1}$ and $\tilde{\omega}_{1}+\tilde{\omega}_{3}$ in the three instances of $S_{\tilde{u}}$ all equal to zero. There is just one way to do this, $\left(\tilde{\omega}_{1}, \tilde{\omega}_{2}, \tilde{\omega}_{3}\right)=(0,0,0)$, and it corresponds to the background peak at the origin. In the second group (BR-I), two out of the three factors include terms in $S_{\tilde{u}}$ while the third one involves a factor in $H_{1}$ or $H_{2}$, which provides another set of 12 combinations. As an example, one of them comprises the first factor in $H_{1}$ and the last two factors in $S_{\tilde{u}}$, which results in the set of equations $-\tilde{\omega}_{1}-\tilde{\omega}_{2}-\tilde{\omega}_{3}= \pm 1, \tilde{\omega}_{1}=0$ and $\tilde{\omega}_{1}+\tilde{\omega}_{3}=0$, which admits the two solutions $\left(\tilde{\omega}_{1}, \tilde{\omega}_{2}, \tilde{\omega}_{3}\right)=(0, \pm 1,0)$. The exercise is repeated for each of the 12 combinations, each of them providing 0,1 or 2 solutions. These peaks fall into the category of mixed background-resonant peaks, after the nomenclature introduced in Denoël |2011. So is it for the third category (BR- $I I$ ) which encompasses the 18 combinations of factors such that only one of them involves a factor in $S_{\tilde{u}}$ whereas the other two involve factors in $H_{1}$ or $H_{2}$. Repeating again the same exercise, it finally yields another list of mixed peaks. At last but not least, the fourth group (R) of peaks gathers the (multi-)resonance peaks. These are obtained by maximizing three out of the four factors in $H_{1}$ or $H_{2}$. Considering for instance the first three factors, the set to be solved reads $-\tilde{\omega}_{1}-\tilde{\omega}_{2}-\tilde{\omega}_{3}= \pm 1, \tilde{\omega}_{1}= \pm 1$ and $\tilde{\omega}_{3}= \pm 1$ which has eight solutions in total. Out of these solutions, some correspond to secondary (triresonant) peaks, such as $\left(\tilde{\omega}_{1}, \tilde{\omega}_{2}, \tilde{\omega}_{3}\right)=(1,-3,1)$, as they provide very small values for the other factors in $t_{\tilde{x}}$ (for this particular example, $H_{2}\left(\tilde{\omega}_{1}+\tilde{\omega}_{2}+\tilde{\omega}_{3},-\tilde{\omega}_{1}-\tilde{\omega}_{3}\right)$ is very small). These peaks are thus discarded.

This analysis provides a list of the important peaks in the trispectrum together with their location and type. Table 1 summarizes the main results of this study and takes an inventory of one background peak in the vicinity of the origin, four plus two mixed background-resonant peaks and four tetra-resonant peaks.

This preliminary analysis was required to apply a Multiple Timescale Spectral Analysis to the determination of the cumulant of the response. To keep the body of the text lighter, we have reported the full developments of the approximation of the fourth order response in Appendix B, and summarized here its expression

$$
\begin{aligned}
\kappa_{4}\left[\tilde{x}_{1}, \tilde{x}_{1}, \tilde{x}_{2}, \tilde{x}_{2}\right]=12 I_{u}^{2} & {\left[1+2 \frac{\pi S_{\tilde{u}}(1)}{2 \xi}\left(1-\frac{\xi_{a}}{2 \xi}\right) \mathcal{I}_{1}(\xi)+\frac{\pi S_{\tilde{u}}(1)}{2 \xi} \mathcal{I}_{2}(\xi)\right.} \\
& \left.-\frac{\xi_{a}}{\xi}\left(1-\frac{\xi_{a}}{2 \xi}\right) \frac{\pi S_{\tilde{u}}(1)}{2 \xi} \mathcal{I}_{3}(\xi)+\frac{1}{4}\left(\frac{\pi S_{\tilde{u}}(1)}{2 \xi}\right)^{2}\left(1-\frac{\xi_{a}}{2 \xi}\right)^{2} \mathcal{I}_{1}(\xi)\right]
\end{aligned}
$$

where

$$
\mathcal{I}_{2}\left(\xi ; S_{\tilde{u}}(\tilde{\omega} ; \alpha)\right)=4 \xi^{2} \iint_{\mathbb{R}^{2}} \frac{S_{\tilde{u}}\left(\tilde{\omega}_{1} ; \alpha\right) S_{\tilde{u}}\left(\tilde{\omega}_{2} ; \alpha\right)}{\left(\tilde{\omega}_{1}-\tilde{\omega}_{2}\right)^{2}+4 \xi^{2}} \mathrm{~d} \tilde{\omega}_{1} \mathrm{~d} \tilde{\omega}_{2}
$$




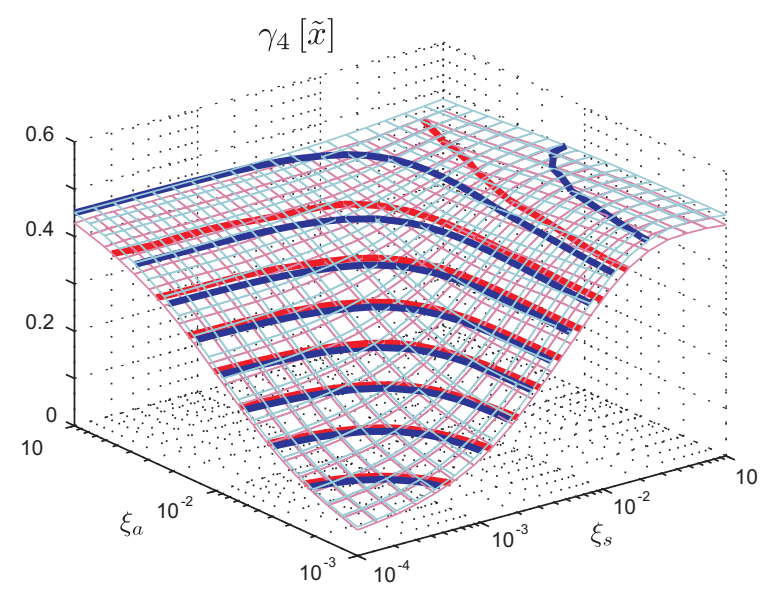

Fig. 7: Comparison of the excess coefficient of the response obtained with the analytical approximation (blue) and with the second Volterra model (red); $I_{u}=0.2, \alpha=0.005$ and $S_{\tilde{u}}(\tilde{\omega} ; \alpha)$ given by (27).

$$
\mathcal{I}_{3}\left(\xi ; S_{\tilde{u}}(\tilde{\omega} ; \alpha)\right)=2 \xi^{2} \iint_{\mathbb{R}^{2}} \frac{\left(\tilde{\omega}_{1} \tilde{\omega}_{2}\left(\left(\tilde{\omega}_{1}-\tilde{\omega}_{2}\right)^{2}+8 \xi^{2}\right)+32 \xi^{4}\right)}{\left(\tilde{\omega}_{1}^{2}+4 \xi^{2}\right)\left(\tilde{\omega}_{2}^{2}+4 \xi^{2}\right)\left(\left(\tilde{\omega}_{1}-\tilde{\omega}_{2}\right)^{2}+4 \xi^{2}\right)} S_{\tilde{u}}\left(\tilde{\omega}_{1} ; \alpha\right) S_{\tilde{u}}\left(\tilde{\omega}_{2} ; \alpha\right) \mathrm{d} \tilde{\omega}_{1} \mathrm{~d} \tilde{\omega}_{2}
$$

have a structure analogous to $\mathcal{I}_{1}$, but are defined in $\mathbb{R}^{2}$. The magnitude of the fourth cumulant of the response is thus governed by the second order resonant-to-background ratio $\beta=\pi S_{\tilde{u}}(1) / 2 \xi$, but also by the integrals $\mathcal{I}_{1}, \mathcal{I}_{2}$ and $\mathcal{I}_{3}$. Accordingly, the excess coefficient is expressed as

$$
\gamma_{4}[\tilde{x}]=12 I_{u}^{2} \frac{1+\left[2\left(1-\frac{\xi_{a}}{2 \xi}\right) \mathcal{I}_{1}(\xi)+\mathcal{I}_{2}(\xi)-\frac{\xi_{a}}{\xi}\left(1-\frac{\xi_{a}}{2 \xi}\right) \mathcal{I}_{3}(\xi)\right] \beta+\frac{1}{4}\left(1-\frac{\xi_{a}}{2 \xi}\right)^{2} \mathcal{I}_{1}(\xi) \beta^{2}}{(1+\beta)^{2}} .
$$

This approximation is represented in Fig. 7, in blue, and compared to the excess coefficient resulting from the Monte Carlo simulation of the second order Volterra model. Again the agreement is remarkable and demonstrates that the timescale separation is well captured with the multiple timescale approach.

\section{Discussion}

We gather in this section a series of remarks, comments and particular cases about the general results obtained above.

\subsection{Integrals $\mathcal{I}_{1}, \mathcal{I}_{2}, \mathcal{I}_{3}$}

The cumulants of the response are expressed as a function of three key integrals, $\mathcal{I}_{k}\left(\xi ; S_{\tilde{u}}(\tilde{\omega} ; \alpha)\right), k=1,2,3$, defined in (57), (61) and 62 . The integral $\mathcal{I}_{1}$ provides the variance of the turbulence fluctuation lowpass filtered by a filter whose cutoff frequency might have the same order of magnitude as the characteristic turbulence frequency. Indeed, at the third and fourth orders, there is an interaction between the two slow timescales of the problem, i.e. the long memory of the structure $\xi^{-1}$ and the slow timescale of the turbulence $\alpha^{-1}$. The integrals $\mathcal{I}_{2}$ and $\mathcal{I}_{3}$ may be interpreted as higher-order lowpass filters of the same kind; they also translate (partial) interactions between these two slow timescales of the problem. When $\alpha \ll \xi$, the lowpass filters select almost the whole frequency content of $\tilde{u}$, reflecting the fact that the memory of the structural system is too short to process the turbulence fluctuation in a dynamic manner. On the contrary, when $\xi \ll \alpha$, the memory of the system is so long that many (symmetrical) oscillations take place during the characteristic timescale of the turbulence and the lowpass filters select a very low frequency range of $\tilde{u}$, reducing therefore the non-Gaussianity of the response. These observations can be formalized more precisely by observing that the PSD given in (27), or other analogous expressions, may be formally written using a scalar function $\varphi(\cdot)$ as

$$
S_{\tilde{u}}(\tilde{\omega} ; \alpha)=\frac{1}{\alpha} \varphi\left(\frac{\tilde{\omega}}{\alpha}\right),
$$



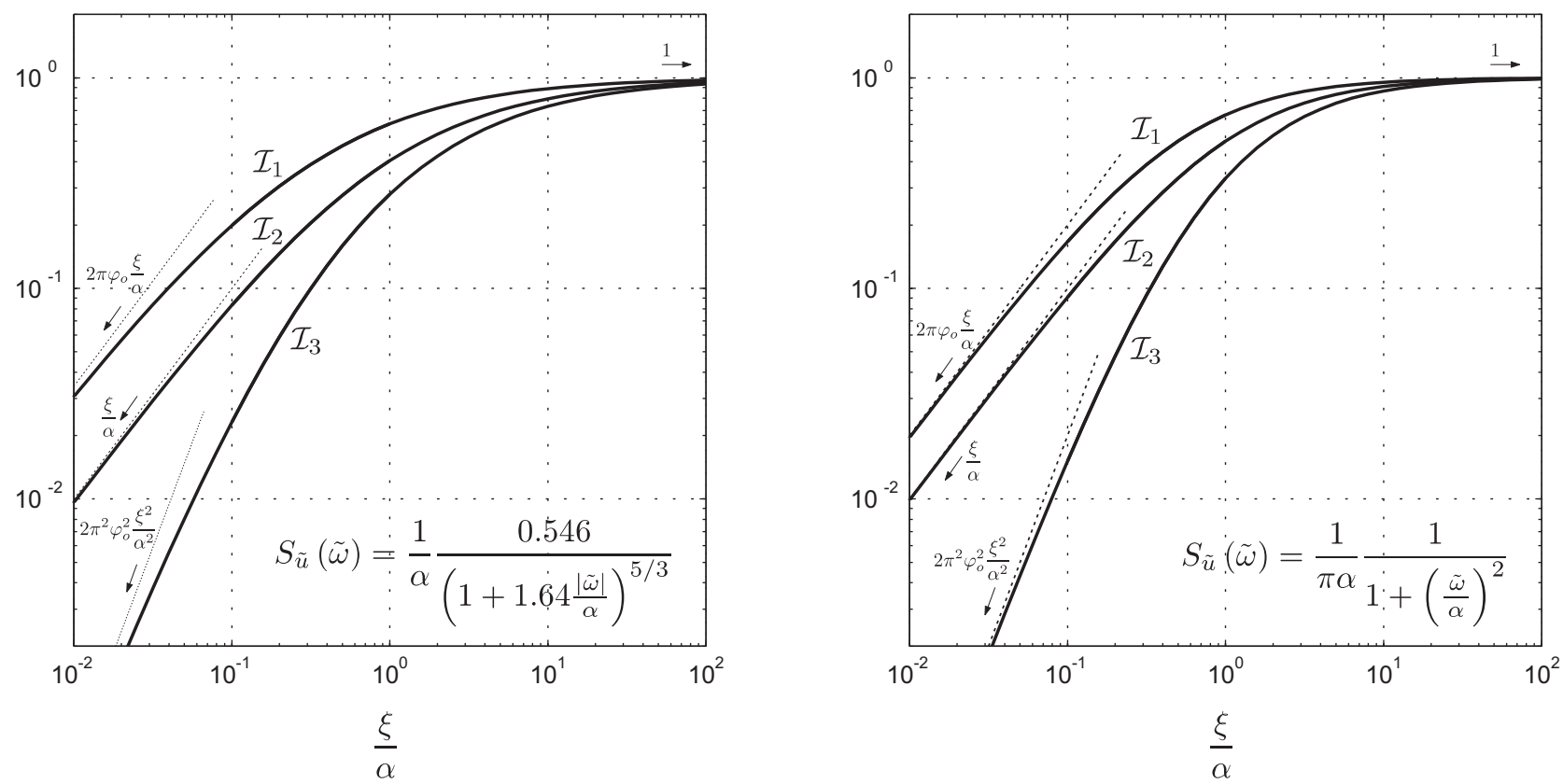

Fig. 8: Integrals $\mathcal{I}_{1}, \mathcal{I}_{2}, \mathcal{I}_{3}$ and their asymptotic behavior, represented for two different power spectral densities of turbulence, (left) a realistic wind turbulence $\varphi_{o}=0.546$ and (right) an Ornstein-Uhlenbeck process $\varphi_{o}=\pi^{-1}$.

Considering this notation, integral $\mathcal{I}_{1}$ becomes

$$
\mathcal{I}_{1}\left(\xi ; S_{\tilde{u}}(\tilde{\omega} ; \alpha)\right)=4 \xi^{2} \int_{\mathbb{R}} \frac{S_{\tilde{u}}(\tilde{\omega} ; \alpha)}{\tilde{\omega}^{2}+4 \xi^{2}} \mathrm{~d} \tilde{\omega}=4 \frac{\xi^{2}}{\alpha^{2}} \int_{\mathbb{R}} \frac{\varphi(\theta)}{\theta^{2}+4 \frac{\xi^{2}}{\alpha^{2}}} \mathrm{~d} \theta
$$

which is a function of only the ratio $\xi / \alpha$, and not $\xi$ and $\alpha$ separately. This statement can be readily extended to integrals $\mathcal{I}_{2}$ and $\mathcal{I}_{3}$. Figure 8 represents these integrals for the power spectral density given in (3) on the left, and for an Ornstein-Uhlenbeck process on the right. Dotted lines represent the asymptotic behaviors, for large and small ratios, i.e. large and small re-scaled cutoff frequencies $\xi / \alpha$. The definitions we chose for those integrals is such that they converge to unity for $\xi / \alpha \gg 1$. Besides, $\mathcal{I}_{1} \rightarrow 2 \pi \xi S_{\tilde{u}}(0 ; \alpha), \mathcal{I}_{2} \rightarrow \xi / \alpha$ and $\mathcal{I}_{3} \rightarrow 2 \pi^{2} \xi^{2} S_{\tilde{u}}^{2}(0 ; \alpha)$ for $\xi / \alpha \ll 1$; these asymptotic behaviors are also reported in Fig. 8 , with $\varphi_{o} \equiv \varphi(0)$.

\subsection{Third order response}

The skewness coefficient given by (58) models the main non-Gaussian feature of the response and its analysis leads to the following observations.

First, for large total damping, the response tends to be quasi-static $(\beta \rightarrow 0)$ and the skewness of the response tends to $3 I_{u}$, i.e. the skewness of the quadratic transformation of the Gaussian process related to the turbulence velocity. Besides, in the very particular case, where (i) $\xi_{a} \ll \xi_{s}$, i.e. the loading is mainly the quadratic transformation of the Gaussian wind velocity field, (ii) $\mathcal{I}_{1} \simeq 1$, i.e. the damping is larger that the dimensionless turbulence frequency $(\alpha \ll \xi)$ and (iii) $\beta \simeq 1$ i.e. the background and resonant contributions to the variance are similar, the skewness of the response might be up to $9 \%$ larger than $3 I_{u}$. This observation results from the maximum of $(1+2 \beta) /(1+\beta)^{3 / 2}$ on $\mathbb{R}^{+}$, equal to $\frac{4}{3} \sqrt{\frac{2}{3}} \simeq 1.089$ for $\beta=\frac{1}{2}$. Quite amazingly, the linear oscillator can thus provide more dissymetry in the output than in the input. This was already observed by Grigoriu and Ariaratnam 1988 andDenoël 2011. However, these three conditions very seldom occur concomitantly and, in usual cases, the response is more Gaussian than a squared Gaussian process $\left(\gamma_{3}[\tilde{x}] \lesssim 3 I_{u}\right)$.

Second, the replacement rule introduced in Section 1, i.e. set $\xi_{a}$ to zero then replace $\xi_{s}$ by $\xi_{a}$, provides the result that would be obtained if the two nonlinear loading terms were neglected. The skewness coefficient of the response has the same expression except that the coefficient $\left(1-\frac{\xi_{a}}{2 \xi}\right)$ in the numerator of 58 disappears. Since $\xi_{a} \leq \xi$ and since other factors in the numerator, namely $\beta$ and $\mathcal{I}_{1}(\xi)$ are positive, neglecting the two nonlinear loading terms 
systematically results in overestimating the skewness of the response. The overestimation factor

$$
\mathcal{O}_{3}=\frac{1+2 \beta \mathcal{I}_{1}(\xi)}{1+2 \beta\left(1-\frac{\xi_{a}}{2 \xi}\right) \mathcal{I}_{1}(\xi)}
$$

indicates that the overestimation (always larger than one) might be as large as a factor of 2 under the simultaneous occurrences of these three conditions: (i) small structural damping $\xi_{s} \ll \xi_{a}$ so that $\xi_{a} \simeq \xi$, (ii) resonant response $\beta \gg 1$ and (iii) $\alpha \ll \xi$, i.e. $\mathcal{I}_{1}(\xi) \simeq 1$.

Third, as the local approximation (53) is linear in $\xi_{a}$, the quadratic structural velocity loading term has actually no influence on the response in the neighborhood of the biresonance peaks. This statement could also be observed by tracing back the origin of the terms in the local approximation and observing that $H_{2, \dot{x}^{2}}$ disappears from this local approximation. This indicates that the above overestimation is the sole consequence of the parametric excitation term. Furthermore, the local approximation in (53) shows that the term proportional to $\xi_{a}$, i.e. related to the parametric excitation term, is negative. It contributes therefore negatively to the skewness of the response while the quadratic transformation of the turbulence always provides a positive contribution. This is another reason why neglecting the parametric excitation term always results in an overestimation of the skewness coefficient of the response.

Fourth, the nonlinear loading terms do not change the signature of the response, compared to the squared turbulence term, as the response is expressed with the same integral $\mathcal{I}_{1}$ as that resulting from the linear structural analysis under a quadratic transformation of a low frequency turbulent excitation [Denoël, 2011].

\subsection{Fourth order response}

The discussion of the excess coefficient given by (63) is rather more complicated as it is a result of different types of mixed background-resonant contributions. However, it provides some simple and important information as well.

First, for large total damping, the response tends to be quasi-static and the excess coefficient of the response tends to $12 I_{u}^{2}$ as for the quadratic transformation of the Gaussian process related to the turbulence velocity. In most usual cases, and especially when the damping coefficient is small, the excess coefficient of the response is smaller than $12 I_{u}^{2}$, as a result of the convolution of the loading and the impulse response function of the structure throughout a long memory lag. However, there are also some very particular cases where the excess coefficient of the response might be larger than the excess coefficient of the loading. For instance, if we notice that the function $\left(1+k_{1} \beta+\left(k_{2} \beta / 2\right)^{2}\right) /(1+\beta)^{2}$ features a maximum equal to $\left(k_{2}^{2}-k_{1}^{2}\right) /\left(4-4 k_{1}+k_{2}^{2}\right)$ for $\beta=2\left(k_{1}-2\right) /\left(k_{2}^{2}-2 k_{1}\right)$, then $\gamma_{4}$ can be slightly larger than unity for $\mathcal{I}_{k} \simeq 1$ with $k \in\{1,2,3\}$ and $\beta \simeq 1$. Again, it is thus possible that the response of the system creates more non-Gaussianity, at fourth order, than what was existing in the squared turbulence process.

Second, invoking again the replacement rule of Section 1, we may determine the overestimation factor of the excess coefficient, that results from neglecting the two nonlinear loading terms.

$$
\mathcal{O}_{4}=\frac{1+\left(2 \mathcal{I}_{1}(\xi)+\mathcal{I}_{2}(\xi)\right) \beta+\frac{1}{4} \mathcal{I}_{1}(\xi) \beta^{2}}{1+\left[2\left(1-\frac{\xi_{a}}{2 \xi}\right) \mathcal{I}_{1}(\xi)+\mathcal{I}_{2}(\xi)-\frac{\xi_{a}}{\xi}\left(1-\frac{\xi_{a}}{2 \xi}\right) \mathcal{I}_{3}(\xi)\right] \beta+\frac{1}{4}\left(1-\frac{\xi_{a}}{2 \xi}\right)^{2} \mathcal{I}_{1}(\xi) \beta^{2}}
$$

There is thus one term (related to $\mathcal{I}_{3}$ ) that is typically specific to the nonlinearity of the loading.

\section{Closing Remarks}

This paper presents approximate solutions of the response of an oscillator subjected to parametric and quadratic velocity excitation, derived by means of a second order Volterra model. After validation of this model in the zone of the parameter space corresponding to small values of the problem parameters, by comparison with Monte Carlo simulations of the original nonlinear system, the cumulants of the response were determined considering a multiple timescale separation technique. This separation considerably simplifies the estimation of the cumulants of the response by dropping by one the order of integration, and results in closed-form solutions for limit values of the parameters. The proposed solutions, however, are not intended to substitute for Monte Carlo simulations, but are rather ment to provide a tool to better understand the dynamic behaviour of a family of nonlinear oscillators subject to random forces. The limitation of the proposed formulation is that the dimensionless parameters of the problem, namely both damping ratios, the turbulence intensity and the characteristic turbulence frequency of the oscillator, be small numbers. 
Among other observations, the proposed formulation (i) confirms the well-known fact that the linear problem provides a very good estimation of the variance of the response, (ii) reveals that the parametric excitation term $u(t) \dot{x}(t)$ might contribute to the skewness and kurtosis by an amount similar to the contribution of the squared turbulence $u^{2}(t)$, but with opposite sign, (iii) shows that the quadratic velocity term $\dot{x}^{2}(t)$ has negligible effects on the skewness of the response, (iv) highlights the existence of complex interactions of the two slow timescales of the problem at third and fourth orders.

\section{Appendix A: Development of the Volterra Frequency Response Functions of the model}

The nondimensional equation of motion (7) representing the given nonlinear system can be formally represented through the block diagram shown in Fig 9 (left) in which the triangles represent gain blocks and $\mathcal{D}^{-1}$ is the inverse of the linear operator representing the left-hand side of $(7)$, i.e.

$$
\mathcal{D}[\tilde{x}]=\tilde{x}^{\prime \prime}+2 \xi_{s} \tilde{x}^{\prime}+\tilde{x}
$$

The ALEs and VFRFs representing this system can be easily obtained by exploiting the topological assemblage technique introduced by Carassale and Kareem [2010]. The first step is to represent each of the blocks composing the system through their Volterra operators and VFRFs. This result is immediate since all the blocks have either a polynomial or differential structure. Then, the representations of the individual blocks are combined to obtain the Volterra operators and the VFRFs of the composite system $\mathcal{H}$. This latter operation can be illustrated as follows. The quantity $f$ (i.e. the input of the operator $\mathcal{D}^{-1}$ ) can be formally obtained following the block diagram from the output $\tilde{x}$ to $f$ along the to two possible branches of the schema. Accordingly, $f$ is the output of the two operators $\mathcal{A}$ and $\mathcal{B}$ represented by the block diagrams reported in Fig. 9 (right) and defined as

$$
\begin{gathered}
f=\mathcal{A}[\tilde{u}]=\mathcal{D}[\tilde{x}]=\mathcal{D}[\mathcal{H}[\tilde{u}]] \\
f=\mathcal{B}[\tilde{u}]=\frac{1}{2 I_{u}}\left[1+I_{u} \tilde{u}-2 \xi_{a} I_{u} \frac{d}{d \tilde{t}} \mathcal{H}[\tilde{u}]\right]^{2}
\end{gathered}
$$

where $\mathcal{H}$ is the (yet unknown) operator providing the system response $\tilde{x}$ given $\tilde{u}$, which we assume can be expanded into a second-order Volterra series as $\mathcal{H}=\mathcal{H}_{0}+\mathcal{H}_{1}+\mathcal{H}_{2}$. Both the operators $\mathcal{A}$ and $\mathcal{B}$ provide $f$ given $\tilde{u}$, thus they must be equal. This obviously implies equalities between their Volterra operators, i.e. $\mathcal{A}_{j}=\mathcal{B}_{j}$ and their VFRFs, i.e. $A_{j}=B_{j}$ at any order $j=\{0,1,2\}$.

The Volterra operators $\mathcal{A}_{j}$ and $\mathcal{B}_{j}$ are calculated from $(69)$ and $(70)$, respectively (see Carassale and Kareem $2010)$, and are equated order by order obtaining the relationships

$$
\begin{gathered}
\mathcal{D}\left[\mathcal{H}_{0}\right]=\frac{1}{2 I_{u}}, \\
\mathcal{D}\left[\mathcal{H}_{1}[\tilde{u}]\right]=\tilde{u}-\xi_{a} \frac{d}{d \tilde{t}} \mathcal{H}_{1}[\tilde{u}], \\
\mathcal{D}\left[\mathcal{H}_{2}[\tilde{u}]\right]=\frac{I_{u}}{2}\left[\tilde{u}-2 \xi_{a} \frac{d}{d \tilde{t}} \mathcal{H}_{1}[\tilde{u}]\right]^{2}-\xi_{a} \frac{d}{d \tilde{t}} \mathcal{H}_{2}[\tilde{u}],
\end{gathered}
$$

which lead to the ALEs (19)-21 once the notation $\tilde{x}_{j}=\mathcal{H}_{j}[\tilde{u}]$ for $j=\{1,2,3\}$ is adopted. These equations can be translated in terms of FRFs as Carassale and Kareem, 2014:

$$
\begin{gathered}
D(0) H_{0}=\frac{1}{2 I_{u}} \\
D(\tilde{\omega}) H_{1}(\tilde{\omega})=1-\xi_{a} i \tilde{\omega} H_{1}(\tilde{\omega}) \\
D\left(\tilde{\omega}_{1}+\tilde{\omega}_{2}\right) H_{2}\left(\tilde{\omega}_{1}, \tilde{\omega}_{2}\right)=\frac{I_{u}}{2}\left[1-2 \xi_{a} i \tilde{\omega}_{1} H_{1}\left(\tilde{\omega}_{1}\right)\right]\left[1-2 \xi_{a} i \tilde{\omega}_{2} H_{1}\left(\tilde{\omega}_{2}\right)\right]-\xi_{a} i\left(\tilde{\omega}_{1}+\tilde{\omega}_{2}\right) H_{2}\left(\tilde{\omega}_{1}, \tilde{\omega}_{2}\right)
\end{gathered}
$$

from which the expressions given in (16) and 17 are readily obtained. 


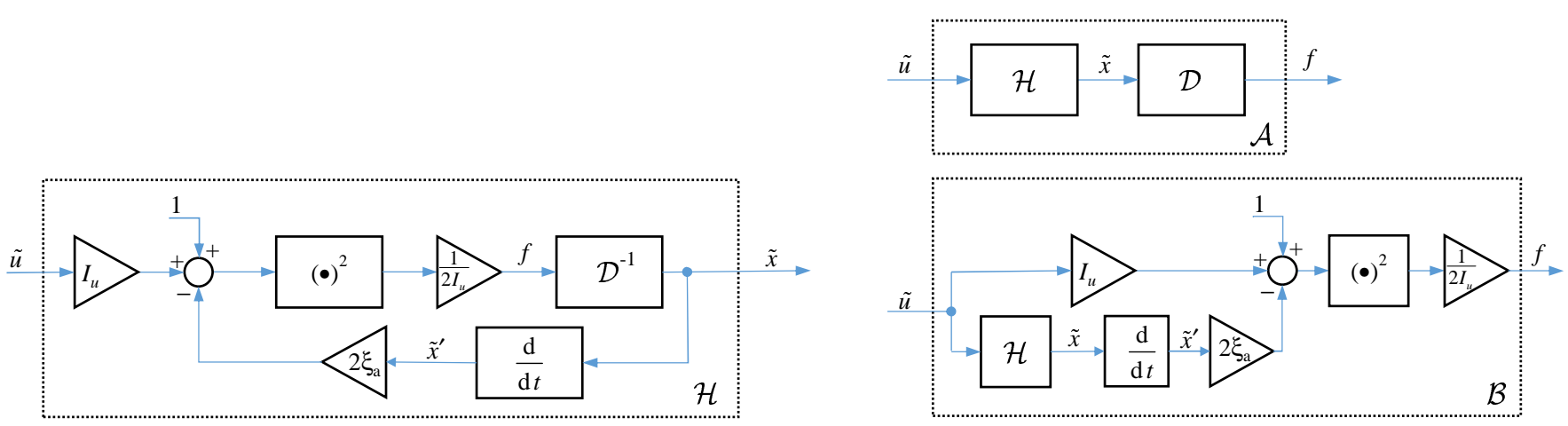

Fig. 9: Block diagram representing the dynamical system (7) (left) and the operators (69) and (70) (right).

\section{Appendix B: Details of the Analytical Solution at order 4}

For one of the twelve terms in the trispectrum, the background component is readily obtained as

$$
I_{u}^{2} \iiint_{\mathbb{R}^{3}} S_{\tilde{u}}\left(\tilde{\omega}_{1}+\tilde{\omega}_{2}+\tilde{\omega}_{3}\right) S_{\tilde{u}}\left(\tilde{\omega}_{1}\right) S_{\tilde{u}}\left(\tilde{\omega}_{1}+\tilde{\omega}_{3}\right) d \tilde{\omega}_{1} d \tilde{\omega}_{2} d \tilde{\omega}_{3}=I_{u}^{2}
$$

so that the total background contribution is given by

$$
\kappa_{4, b}\left[\tilde{x}_{1}, \tilde{x}_{1}, \tilde{x}_{2}, \tilde{x}_{2}\right]=12 I_{u}^{2} .
$$

With no approximation, the remainder is expressed as

$$
\begin{gathered}
\rho_{4}=\iiint_{\mathbb{R}^{3}}\left[4 H_{1}\left(-\tilde{\omega}_{1}-\tilde{\omega}_{2}-\tilde{\omega}_{3}\right) H_{1}\left(\tilde{\omega}_{1}\right) H_{2}\left(-\tilde{\omega}_{1}, \tilde{\omega}_{1}+\tilde{\omega}_{3}\right) H_{2}\left(\tilde{\omega}_{1}+\tilde{\omega}_{2}+\tilde{\omega}_{3},-\tilde{\omega}_{1}-\tilde{\omega}_{3}\right)-I_{u}^{2}\right] \\
S_{\tilde{u}}\left(\tilde{\omega}_{1}+\tilde{\omega}_{2}+\tilde{\omega}_{3}\right) S_{\tilde{u}}\left(\tilde{\omega}_{1}\right) S_{\tilde{u}}\left(\tilde{\omega}_{1}+\tilde{\omega}_{3}\right) d \tilde{\omega}_{1} d \tilde{\omega}_{2} d \tilde{\omega}_{3} .
\end{gathered}
$$

Follows then a series of coordinate stretching and local approximations to derive the successive contributions to the integral. The first group BR-I of mixed background/resonant contribution is analyzed by focusing on the peak located at $(-1,0,1)$ with the new coordinates defined as

$$
\tilde{\omega}_{1}=1+\xi \eta_{1} \quad ; \quad \tilde{\omega}_{2}=\xi \eta_{2} \quad ; \quad \tilde{\omega}_{3}=-1+\xi \eta_{3} .
$$

Substituting these new coordinates in the brackets of $(79)$, then disregarding second order terms in $\xi$ as well as terms that become negligible outside the unit disk $\|\boldsymbol{\eta}\| \lesssim 1$, yields

$$
\rho_{4, b r I}=I_{u}^{2} \iiint_{\mathbb{R}^{3}} \frac{\left(\mathrm{i}+\eta_{1}\right) \xi-\mathrm{i} \xi_{a}}{4\left(1+\eta_{1}^{2}\right)\left(\mathrm{i}-\eta_{3}\right) \xi^{3}} S_{\tilde{u}}\left(\xi\left(\eta_{1}+\eta_{2}+\eta_{3}\right)\right) S_{\tilde{u}}\left(1+\xi \eta_{1}\right) S_{\tilde{u}}\left(\xi\left(\eta_{1}+\eta_{3}\right)\right) \xi^{3} \mathrm{~d} \eta_{1} \mathrm{~d} \eta_{2} \mathrm{~d} \eta_{3},
$$

i.e. a crest-like approximation of the frequency response function, where the fraction is well integrable in the far field, along $\eta_{1}$ and $\eta_{3}$. A first explicit integration with respect to $\eta_{2}$ might be carried out. Then assuming $S_{\tilde{u}}\left(1+\xi \eta_{1}\right) \simeq S_{\tilde{u}}(1)$ over the unit disk yields

$$
\rho_{4, b r I}=I_{u}^{2} S_{\tilde{u}}(1) \iint_{\mathbb{R}^{2}} \frac{\left(\mathrm{i}+\eta_{1}\right) \xi-\mathrm{i} \xi_{a}}{4\left(1+\eta_{1}^{2}\right)\left(\mathrm{i}-\eta_{3}\right) \xi^{3}} S_{\tilde{u}}\left(\xi\left(\eta_{1}+\eta_{3}\right)\right) \xi^{2} \mathrm{~d} \eta_{1} \mathrm{~d} \eta_{3},
$$

which might be further simplified as $S_{\tilde{u}}\left(\xi\left(\eta_{1}+\eta_{3}\right)\right)$ only varies along the gradient $\nabla\left(\eta_{1}+\eta_{3}\right)$. Introducing the unit-Jacobian change of variable

$$
\left(\begin{array}{l}
\vartheta_{1} \\
\vartheta_{2} \\
\vartheta_{3}
\end{array}\right)=\left[\begin{array}{lll}
1 & 0 & 0 \\
1 & 1 & 1 \\
1 & 0 & 1
\end{array}\right]\left(\begin{array}{l}
\eta_{1} \\
\eta_{2} \\
\eta_{3}
\end{array}\right)
$$


yields

$$
\rho_{4, b r I}=I_{u}^{2} S_{\tilde{u}}(1) \iint_{\mathbb{R}^{2}} \frac{\left(\mathrm{i}+\vartheta_{1}\right) \xi-\mathrm{i} \xi_{a}}{4\left(1+\vartheta_{1}^{2}\right)\left(\mathrm{i}+\vartheta_{1}-\vartheta_{3}\right) \xi^{3}} S_{\tilde{u}}\left(\xi \vartheta_{3}\right) \xi^{2} \mathrm{~d} \vartheta_{1} \mathrm{~d} \vartheta_{3},
$$

whose real part, of interest, is explicitly integrated along $\vartheta_{1}$,

$$
\rho_{4, b r I}=\frac{\pi}{2} I_{u}^{2} S_{\tilde{u}}(1) \frac{2 \xi_{s}+\xi_{a}}{\xi} \int_{\mathbb{R}} \frac{S_{\tilde{u}}\left(\xi \vartheta_{3}\right)}{4+\vartheta_{3}^{2}} \mathrm{~d} \vartheta_{3},
$$

Multiplying this expression by 4 to take into consideration the four BR-(I) peaks then 12 for the twelve terms, and reverting back to the original frequency space, the contribution of the BR-(I) peaks to the fourth cumulant reads

$$
\kappa_{4, b r I}\left[\tilde{x}_{1}, \tilde{x}_{1}, \tilde{x}_{2}, \tilde{x}_{2}\right]=6 I_{u}^{2} \pi S_{\tilde{u}}(1)\left(2 \xi_{s}+\xi_{a}\right) \mathcal{I}_{1}(\xi)
$$

with the same $\mathcal{I}_{1}(\xi)$ as defined in (57). This contribution to the fourth cumulant features only two factors in $S_{\tilde{u}}(1)$, while they were three at the beginning. The reason is because of the crest-like shape of the local approximation, the extent of the crest being governed by the ratio $\alpha / \xi$. For these reasons, this first contribution is very similar to the biresonant contribution to the third cumulant. In fact, $\kappa_{4, b r, I}=I_{u} \kappa_{3, r}\left[\tilde{x}_{1}, \tilde{x}_{1}, \tilde{x}_{2}\right]$.

The coordinates

$$
\tilde{\omega}_{1}=\xi \eta_{1} \quad ; \quad \tilde{\omega}_{2}=-1+\xi \eta_{2} \quad ; \quad \tilde{\omega}_{3}=1+\xi \eta_{3}
$$

are introduced to focus on one of the two BR-(II) peaks. Plugging this stretching into (79), the original expression of $\rho_{4}$-which is allowed since there are no overlapping of the different contributions in their stretched space-, becomes

$\rho_{4, b r I I}=I_{u}^{2} \iiint_{\mathbb{R}^{3}} \frac{\xi^{2}\left(\eta_{1}^{2}+2 \eta_{1} \eta_{3}+\eta_{3}^{2}+1\right)+\xi_{a}^{2}-2 \xi_{a} \xi}{4\left(\mathrm{i}-\eta_{2}\right)\left(\eta_{3}-\mathrm{i}\right)\left(1+\left(\eta_{1}+\eta_{3}\right)^{2}\right) \xi^{4}} S_{\tilde{u}}\left(\xi\left(\eta_{1}+\eta_{2}+\eta_{3}\right)\right) S_{\tilde{u}}\left(\xi \eta_{1}\right) S_{\tilde{u}}\left(1+\xi\left(\eta_{1}+\eta_{3}\right)\right) \xi^{3} \mathrm{~d} \eta_{1} \mathrm{~d} \eta_{2} \mathrm{~d} \eta_{3}$,

after conserving the first order terms in $\xi$ and $\xi_{a}$. Recoursing again to the same change of variable 83 and observing that the last factor in the integrand $S_{\tilde{u}}\left(1+\xi \vartheta_{3}\right) \simeq S_{\tilde{u}}(1)$ on the $\vartheta$-unit disk, integration along $\vartheta_{3}$ may be carried out explicitly, which leaves

$\rho_{4, b r I I}=I_{u}^{2} S_{\tilde{u}}(1) \frac{\pi}{4} \xi^{3} \iint_{\mathbb{R}^{2}}\left[\frac{4}{\left(\left(\vartheta_{1}-\vartheta_{2}\right)^{2}+4\right) \xi^{2}}-\frac{\left(\vartheta_{1} \vartheta_{2}\left(\left(\vartheta_{1}-\vartheta_{2}\right)^{2}+8\right)+32\right) \xi_{a}\left(2 \xi-\xi_{a}\right)}{\left(\vartheta_{1}^{2}+4\right)\left(\vartheta_{2}^{2}+4\right)\left(\left(\vartheta_{1}-\vartheta_{2}\right)^{2}+4\right) \xi^{4}}\right] S_{\tilde{u}}\left(\xi \vartheta_{2}\right) S_{\tilde{u}}\left(\xi \vartheta_{1}\right) \mathrm{d} \vartheta_{1} \mathrm{~d} \vartheta_{2}$.

Reverting back to the original frequency space and multiplying by two in order to account for both BR-(II) peaks, we have

$$
\kappa_{4, b r I I}\left[\tilde{x}_{1}, \tilde{x}_{1}, \tilde{x}_{2}, \tilde{x}_{2}\right]=12 I_{u}^{2} \frac{\pi S_{\tilde{u}}(1)}{2 \xi}\left[\mathcal{I}_{2}\left(\xi ; S_{\tilde{u}}(\tilde{\omega} ; \alpha)\right)-\frac{\xi_{a}\left(2 \xi_{s}+\xi_{a}\right)}{2 \xi^{2}} \mathcal{I}_{3}\left(\xi ; S_{\tilde{u}}(\tilde{\omega} ; \alpha)\right)\right]
$$

where

$$
\begin{aligned}
& \mathcal{I}_{2}\left(\xi ; S_{\tilde{u}}(\tilde{\omega} ; \alpha)\right)=4 \xi^{2} \iint_{\mathbb{R}^{2}} \frac{S_{\tilde{u}}\left(\tilde{\omega}_{1} ; \alpha\right) S_{\tilde{u}}\left(\tilde{\omega}_{2} ; \alpha\right)}{\left(\tilde{\omega}_{1}-\tilde{\omega}_{2}\right)^{2}+4 \xi^{2}} \mathrm{~d} \tilde{\omega}_{1} \mathrm{~d} \tilde{\omega}_{2} \\
& \mathcal{I}_{3}\left(\xi ; S_{\tilde{u}}(\tilde{\omega} ; \alpha)\right)=2 \xi^{2} \iint_{\mathbb{R}^{2}} \frac{\left(\tilde{\omega}_{1} \tilde{\omega}_{2}\left(\left(\tilde{\omega}_{1}-\tilde{\omega}_{2}\right)^{2}+8 \xi^{2}\right)+32 \xi^{4}\right)}{\left(\tilde{\omega}_{1}^{2}+4 \xi^{2}\right)\left(\tilde{\omega}_{2}^{2}+4 \xi^{2}\right)\left(\left(\tilde{\omega}_{1}-\tilde{\omega}_{2}\right)^{2}+4 \xi^{2}\right)} S_{\tilde{u}}\left(\tilde{\omega}_{1} ; \alpha\right) S_{\tilde{u}}\left(\tilde{\omega}_{2} ; \alpha\right) \mathrm{d} \tilde{\omega}_{1} \mathrm{~d} \tilde{\omega}_{2}
\end{aligned}
$$

Finally, the tetraresonant contribution is developed by introducing the stretched coordinates

$$
\tilde{\omega}_{1}=-1+\xi \eta_{1} \quad ; \quad \tilde{\omega}_{2}=-1+\xi \eta_{2} \quad ; \quad \tilde{\omega}_{3}=1+\xi \eta_{3}
$$

which allow focusing specifically on one out of the four tetraresonant peaks. Pursuing the same exercise as above, and limiting the expression of the product of frequency Volterra kernels to a local approximation, we have

$$
\begin{aligned}
\rho_{4, r}=I_{u}^{2} \iiint_{\mathbb{R}^{3}} \frac{-2\left(\eta_{1}+\mathrm{i}\right)\left(\eta_{1}+\eta_{2}+\eta_{3}-\mathrm{i}\right) \xi^{2}+2 \mathrm{i} \xi_{a} \xi\left(\eta_{2}+\eta_{3}-2 \mathrm{i}\right)-2 \xi_{a}^{2}}{32\left(1+\eta_{1}^{2}\right)\left(\eta_{2}-\mathrm{i}\right)\left(\eta_{3}-\mathrm{i}\right)\left(1+\left(\eta_{1}+\eta_{2}+\eta_{3}\right)^{2}\right) \xi^{6}} \\
S_{\tilde{u}}\left(-1+\xi\left(\eta_{1}+\eta_{2}+\eta_{3}\right)\right) S_{\tilde{u}}\left(-1+\xi \eta_{1}\right) S_{\tilde{u}}\left(\xi\left(\eta_{1}+\eta_{3}\right)\right) \xi^{3} \mathrm{~d} \eta_{1} \mathrm{~d} \eta_{2} \mathrm{~d} \eta_{3}
\end{aligned}
$$


Approximating the first two factors in $S_{\tilde{u}}$ by a constant value and considering the change of variable (83) yields

$$
\begin{aligned}
\rho_{4, r} & =I_{u}^{2} \xi^{3} S_{\tilde{u}}^{2}(1) \int_{\mathbb{R}} \frac{\left(\xi_{a}-\left(1-\mathrm{i} \vartheta_{1}\right) \xi\right)\left(\xi_{a}-\left(1+\mathrm{i} \vartheta_{2}\right) \xi\right)}{16\left(1+\vartheta_{1}^{2}\right)\left(1+\vartheta_{2}^{2}\right)\left(\mathrm{i}+\vartheta_{1}-\vartheta_{3}\right)\left(-\mathrm{i}+\vartheta_{2}-\vartheta_{3}\right) \xi^{6}} S_{\tilde{u}}\left(\xi \vartheta_{3}\right) \mathrm{d} \vartheta_{3} \\
& =\frac{\pi^{2}}{16} I_{u}^{2} \xi^{3} \frac{S_{\tilde{u}}^{2}(1)}{\xi^{2}} \frac{\left(2 \xi_{s}+\xi_{a}\right)^{2}}{\xi^{2}} \int_{\mathbb{R}} \frac{S_{\tilde{u}}\left(\xi \vartheta_{3}\right)}{4 \xi^{2}+\xi^{2} \vartheta_{3}^{2}} \mathrm{~d} \vartheta_{3} .
\end{aligned}
$$

Multiplication of this result by four, in order to take into account the four tetraresonant peaks, then 12 again for the twelve factors, and application of the mapping reverting back to the original physical space finally yields

$$
\kappa_{4, r}\left[\tilde{x}_{1}, \tilde{x}_{1}, \tilde{x}_{2}, \tilde{x}_{2}\right]=3 I_{u}^{2} \frac{\pi^{2} S_{\tilde{u}}^{2}(1)}{4 \xi^{2}}\left(\frac{\xi_{s}+\frac{\xi_{a}}{2}}{\xi_{s}+\xi_{a}}\right)^{2} \mathcal{I}_{1}\left(\xi ; S_{\tilde{u}}\right)
$$

where $\mathcal{I}_{1}$ was defined in 57 .

$$
\mathcal{I}_{1}\left(\xi ; S_{\tilde{u}}(\tilde{\omega} ; \alpha)\right)=4 \xi^{2} \int_{\mathbb{R}} \frac{S_{\tilde{u}}(\tilde{\omega} ; \alpha)}{\tilde{\omega}^{2}+4 \xi^{2}} \mathrm{~d} \tilde{\omega}
$$

In summary, the total cumulant is expressed as the sum of a background component, two mixed backgroundresonant components and a (tetra-)resonant component.

\section{References}

E. Bedrosian and S.O. Rice. The output properties of volterra systems (nonlinear systems with memory) driven by harmonic and gaussian inputs. Proceedings of the IEEE, 59(12):1688-1707, Dec 1971.

S. Benfratello and G. Muscolino. Filter approach to the stochastic analysis of mdof wind-excited structures. Probabilistic engineering mechanics, 14(4):311-321, 1999.

S. Benfratello, G. Falsone, and G. Muscolino. Influence of the quadratic term in the alongwind stochastic response of sdof structures. Engineering Structures, 18(9):685-695, 1996.

S. Benfratello, M. Di Paola, and P.D. Spanos. Stochastic response of mdof wind-excited structures by means of volterra series approach. Journal of Wind Engineering and Industrial Aerodynamics, 74:1135-1145, 1998.

A. Buss and M. Grigoriu. Reliability of linear oscillators subject to wind loads. Journal of Wind Engineering and Industrial Aerodynamics, 36:571-577, 1990.

L. Carassale and A. Kareem. Modeling nonlinear systems by volterra series. Journal of Engineering Mechanics, 136(6):801-818, 2010.

L. Carassale and A. Kareem. Synthesis of multi-variate volterra systems by a topological assemblage scheme. Probabilistic Engineering Mechanics, 37:109-122, 2014.

M C Constantinou and A S Papageorgiou. Stochastic response of practical sliding isolation systems. Probabilistic Engineering Mechanics, 5(1):27-34, 1990. ISSN 0266-8920.

A.G. Davenport. The application of statistical concepts to the wind loading of structures. Proceedings of the Institute of Civil Engineers, 19:449-472, 1961.

V Denoël. Estimation of modal correlation coefficients from background and resonant responses. Structural Engineering and Mechanics: an International Journal, 32(6):725-740, 2009.

V. Denoël. On the background and biresonant components of the random response of single degree-of-freedom systems under non-gaussian random loading. Engineering Structures, 33(8):2271-2283, 2011.

V. Denoël. Multiple timescale spectral analysis. Probabilistic Engineering Mechanics, 39:69-86, 2015.

M. Di Paola. Digital simulation of wind field velocity. Journal of Wind Engineering and Industrial Aerodynamics, 74:91-109, 1998. 
M. Di Paola, G. Falsone, and A. Pirrotta. Stochastic response analysis of nonlinear systems under gaussian inputs. Probabilistic engineering mechanics, 7(1):15-21, 1992.

C. Floris, M. Pastorino, and M.C. Sandrelli. Stochastic along-wind response of point-like structures. Journal of wind engineering and industrial aerodynamics, 89(2):165-185, 2001.

M. Grigoriu. Response of linear systems to quadratic gaussian excitations. journal of Engineering Mechanics, 112 (6):523-535, 1986.

M. Grigoriu and S.T. Ariaratnam. Response of linear systems to polynomials of gaussian processes. Journal of applied mechanics, 55(4):905-910, 1988.

I. Gullo, G. Muscolino, and M. Vasta. Non-gaussian probability density function of sdof linear structures under wind actions. Journal of Wind Engineering and Industrial Aerodynamics, 74:1123-1134, 1998.

K.R. Gurley, M.A. Tognarelli, and A. Kareem. Analysis and simulation tools for wind engineering. Probabilistic Engineering Mechanics, 12(1):9-31, 1997.

V. Gusella and A.L. Materazzi. Non-gaussian along-wind response analysis in time and frequency domains. Engineering Structures, 22(1):49-57, 2000.

M. Kac and A.J.F. Siegert. On the theory of noise in radio receivers with square law detectors. Journal of Applied Physics, 18(4):383-397, 1947.

A. Kareem. Nonlinear wind velocity term and response of compliant offshore structures. Journal of Engineering Mechanics, 110(10):1573-1578, 1984.

A. Kareem. Wind effects on structures: a probabilistic viewpoint. Probabilistic Engineering Mechanics, 2(4): 166-200, 1987.

A. Kareem and J. Zhao. Analysis of non-gaussian surge response of tension leg platforms under wind loads. Journal of Offshore Mechanics and Arctic Engineering, 116(3):137-144, 1994.

A. Kareem, M.A. Tognarelli, and K.R. Gurley. Modeling and analysis of quadratic term in the wind effects on structures. Journal of Wind Engineering and Industrial Aerodynamics, 74:1101-1110, 1998.

X. Li, S. Quek, and C. Koh. Stochastic response of offshore platforms by statistical cubicization. Journal of Engineering Mechanics, 121(10):1056-1068, 1995.

A. Papoulis and S.U. Pillai. Probability, random variables, and stochastic processes. Tata McGraw-Hill Education, 2002.

M. Schetzen. The Volterra and Wiener theories of nonlinear systems. John Wiley \& Sons, 1980.

C. Soize. Gust loading factors with nonlinear pressure terms. Journal of Structural Division-ASCE, 104(6), 1978.

G. Solari and G. Piccardo. Probabilistic 3-d turbulence modeling for gust buffeting of structures. Probabilistic Engineering Mechanics, 16(1):73-86, 2001.

J.A. Vazquez Feijoo, K. Worden, and R. Stanway. Associated linear equations for volterra operators. Mechanical Systems and Signal Processing, 19(1):57-69, 2005. 